\title{
Estimation of Stress-Strength Reliability for Multicomponent System with Rayleigh Data
}

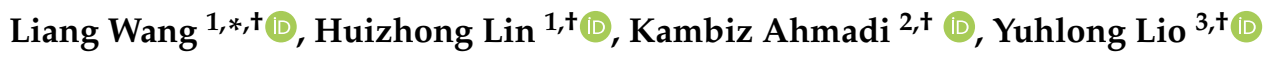 \\ 1 School of Mathematics, Yunnan Normal University, Kunming 650500, China; huizhonglin@user.ynnu.edu.cn \\ 2 Department of Computer Science, Faculty of Mathematical Sciences, Shahrekord University, \\ Shahrekord 115, Iran; K.Ahmadi@sku.ac.ir \\ 3 Department of Mathematical Sciences, University of South Dakota, Vermillion, SD 57069, USA; \\ yuhlong.lio@usd.edu \\ * Correspondence: wangliang@ynnu.edu.cn or liang610112@163.com \\ + These authors contributed equally to this work.
}

Citation: Wang, L.; Lin, H.; Ahmadi, K.; Lio, Y. Estimation of Stress-Strength Reliability for Multicomponent System with Rayleigh Data. Energies 2021, 14, 7917. https://doi.org/10.3390/en14237917

Academic Editors: Yu Liu, Zequn Wang and Jinhua Mi

Received: 10 November 2021 Accepted: 22 November 2021 Published: 25 November 2021

Publisher's Note: MDPI stays neutral with regard to jurisdictional claims in published maps and institutional affiliations.

Copyright: (c) 2021 by the authors. Licensee MDPI, Basel, Switzerland. This article is an open access article distributed under the terms and conditions of the Creative Commons Attribution (CC BY) license (https:/ / creativecommons.org/licenses/by/ $4.0 /)$.

\begin{abstract}
Inference is investigated for a multicomponent stress-strength reliability (MSR) under Type-II censoring when the latent failure times follow two-parameter Rayleigh distribution. With a context that the lifetimes of the strength and stress variables have common location parameters, maximum likelihood estimator of MSR along with the existence and uniqueness is established. The associated approximate confidence interval is provided via the asymptotic distribution theory and delta method. Meanwhile, alternative generalized pivotal quantities-based point and confidence interval estimators are also constructed for MSR. More generally, when the lifetimes of strength and stress variables follow Rayleigh distributions with unequal location parameters, likelihood and generalized pivotal-based estimators are provided for MSR as well. In addition, to compare the equivalence of different strength and stress parameters, a likelihood ratio test is provided. Finally, simulation studies and a real data example are presented for illustration.
\end{abstract}

Keywords: multicomponent stress-strength model; rayleigh distribution; likelihood estimation; generalized pivotal estimation; asymptotic theory

\section{Introduction and Notation}

Stress-strength model plays a substantial role in lifetime studies and engineering applications, where the system survives if its strength is greater than a certain amount of stress imposed. The stress-strength reliability (SSR) parameter $R$ is defined as $R=P(Y<X)$, where $X$ represents the random strength of the system and $Y$ is the random stress applied to the system. In the last few years, the stress-strength model has been widely used in various fields such as reliability engineering, seismology, hydrology, economics, and survival analysis among others, and inference for SSR has been discussed by many authors. See, for example, the works of by Eryilmaz [1], Kundu and Raqad [2], Krishnamoorthy and Lin [3], Mokhlis et al. [4], and Wang et al. [5]. In a conventional study, inference for SSR has focused on the system with a sole component. However, many systems are composed of multiple components to achieve their functions. Therefore, the SSR has been extended to the case of a multicomponent system. There are many classical multicomponent systems in practice, and some classical ones include a series system, parallel system, or combinations of these two systems. Generally, a multicomponent system consists of $k$ independent and identically distributed (i.i.d.) strength components with a common stress, and the system functions when at least $s(1 \leq s \leq k)$ components simultaneously survive. In literature, this system is referred to as the s-out-of- $k$ : $G$ system, which includes a conventional series system and parallel system as its special cases. In reliability engineering and lifetime studies, many examples can be viewed as multicomponent systems. For example, in a communication system with three transmitters, the average message load may be such that at least two 
transmitters must be operational at all times otherwise critical messages may be lost. Thus, the transmission subsystem functions as a 2-out-of-3: G system. Another example in the aircraft industry is where the Airbus A-380 has four engines and the airplane is capable of flying if and only if at least two of its four engines are functioning, is referred as a 2-out-of-4 $41 \mathrm{G}$ system.

For the s-out-of- $k \mathrm{G}$ multicomponent system, suppose the $k$ components of the system have strengths following $k$ i.i.d. random variables, $X_{1}, X_{2}, \ldots, X_{k}$, with the common cumulative distribution function $(\mathrm{CDF}), F(\cdot)$, and each component experiences a random stress $Y$ of CDF $G(\cdot)$. Following Bhattacharyya and Johnson [6], the multicomponent stress-strength reliability (MSR): parameter $R_{s, k}$ is given by

$$
\begin{aligned}
R_{s, k} & =P\left(\text { at least } s \text { of the }\left(X_{1}, X_{2}, \ldots, X_{k}\right) \text { exceed } Y\right) \\
& =\sum_{i=s}^{k}\left(\begin{array}{c}
k \\
i
\end{array}\right) \int_{-\infty}^{\infty}[1-F(t)]^{i}[F(t)]^{k-i} d G(t) .
\end{aligned}
$$

The 1s-out-of- $k$ G system has raised much attention and the associated reliability estimation of the multicomponent system has been widely discussed by many researches. For instance, Dey et al. [7], Kayal [8], Kizilaslan [9,10], Kizilaslan and Nadar [11], Nadar and Kizilaslan [12], and Rao et al. [13], when the underlying distributions follow Kumaraswamy, Chen, a class of inverse exponentiated, proportional reversed hazard rate model, bivariate Kumaraswamy, bivariate Weibull, bathtub-shaped, and Burr XII, respectively.

The Rayleigh distribution is widely applied to model an event in reliability because of its linear and increasing failure rate function. Let $T$ be the random variable of Rayleigh distribution, whose CDF and probability density function (PDF) are given by:

$$
F(t ; \lambda, \alpha)=1-e^{-\lambda(t-\alpha)^{2}} \quad \text { and } \quad f(t ; \lambda, \alpha)=2 \lambda(t-\alpha) e^{-\lambda(t-\alpha)^{2}}, t>\alpha,
$$

where $\lambda>0$ and $\alpha>0$ are the rate and location parameters, respectively. Hereafter, Rayleigh distribution with parameters $\alpha$ and $\lambda$ will be denoted by $\operatorname{RL}(\lambda, \alpha) \cdot \operatorname{RL}(\lambda, 0)$ was initially introduced by Rayleigh [14] in acoustics problems related to the studies of physics. The extended $\operatorname{RL}(\lambda, \alpha)$ by inserting the location parameter $\alpha \geq 0$ provides more flexibility of the probability modeling on date observations that could have positive support. Since then, it has been shown as an important distribution modeling for skewed datasets. The structural properties of this distribution are related to some common distributions such as gamma, Weibull, chi-square, Rice, and extreme value models. Several authors have discussed Rayleigh distribution under different cases. For example, some recent contributions by Ahrari et al. [15], Dey et al. [16], Fundi et al. [17], and Ghani and Isa [18].

In statistical inference, sample size is an important issue in data analysis, which sometimes has such a strong effect on the validity of the associated results. Since modern products always feature high reliable and a long life cycle, it is frequently impossible to obtain complete failure times for all test units in practice, the datasets collected often lack sufficient samples, and the associated conventional likelihood-based inferential results may not have targeted statistical properties. Motivated by previous reasons, the main goal of this study is to discuss different inferential methods for MSR $R_{s, k}$ when the latent strength and stress random variables have common and unequal Rayleigh parameters. The main contributions of this paper are presented as follows. Firstly, when the latent strength and stress failure times follow two-parameter Rayleigh distributions, the multicomponent stress-strength model is considered under censored data scenario. Secondly, under common and unequal location parameter cases, the existence and uniqueness of maximum likelihood estimators of the unknown strength and stress parameters are established, and the associated estimates for stress-strength reliability are obtained in consequence. Finally, comparing with traditional likelihood-based estimation, alternative novelty generalized estimates for MSR are proposed where the performance of the proposed generalized method appears superior than the classical likelihood results. In addition, after a comprehensive lit- 
erature retrieval, it is worth mentioning that the estimation of the multicomponent Rayleigh stress-strength model was also discussed by Rao [19], and some difference between the aforementioned and our paper should be discriminated here for clarity. In Rao's [19] discussion, the author has just provided the maximum likelihood and moment estimations of the reliability for a multicomponent system when the latent stress and strength lifetimes follow one-parameter Rayleigh under complete data. Whereas, in our studies the innovative difference lies in that the results were extended to the multicomponent stress-strength model based on two-parameter Rayleigh distribution under censored data. Furthermore, as mentioned above, besides the likelihood-based estimators with the existence and uniqueness for strength and stress parameters established, alternative generalized point and interval estimates are also proposed for comparison. In addition, previous conventional likelihood and generalized inferential approaches are both discussed under common and unequal strength and stress parameters cases, respectively. Furthermore, unlike the natural way for computing maximum likelihood estimators via taking direct derivatives for likelihood equation in Rao's [19] work, a profile likelihood approach is used for maximum likelihood estimation in our discussion for two-parameter Rayleigh models, which is more efficient and concise in a computational procedure. Therefore, comparing with Rao's [19] work, there are significant differences from the perspectives of both inferential methods and lifetime models in these two papers. To the best of our knowledge, no work has been carried out to study the pivotal quantities-based generalized estimates for MSR parameter $R_{s, k}$ based on two-parameter Rayleigh strength and stress distributions when the failure times are obtained under Type-II censoring.

This rest of this paper is organized as follows. In Section 2, the strength and stress samples are described, and the likelihood function is given. Section 3 presents different inferential approaches to estimate MSR parameter $R_{s, k}$ when latent strength and stress lifetime models have a common parameter. Correspondingly, estimation is also discussed based on unequal Rayleigh parameters in Section 4. In order to compare the equivalence of the strength and stress location parameters, a testing problem of interested parameters is presented in Section 5. Simulation studies and a real data example are provided in Section 6 for illustration. Finally, Section 7 gives some concluding remarks.

\section{Model Description and Likelihood Function}

Suppose that $n s$-out-of- $k \mathrm{G}$ systems are put on a life-test experiment, each system contains $k$ i.i.d. strength components subject to a common stress. Based on the failure mechanism of the s-out-of- $k$ G system, failure samples for strength and stress variables are observed as follows,

$$
\begin{aligned}
& \text { Observed strength variables } \\
& \left(\begin{array}{cccc}
X_{11} & X_{12} & \cdots & X_{1 s} \\
\vdots & \vdots & \ddots & \vdots \\
X_{n 1} & X_{n 2} & \cdots & X_{n s}
\end{array}\right) \text { and }\left(\begin{array}{c}
Y_{1} \\
\vdots \\
Y_{n}
\end{array}\right)
\end{aligned}
$$

where $\left\{X_{i 1}, X_{i 2}, \ldots, X_{i s}\right\}$ are independent first $s$ strength samples for the $i$ th system and $Y_{i}$ is the associated common stress variable, $i=1,2, \ldots, n$. Let the lifetimes of the i.i.d. system components be distributed according to $\operatorname{CDF} F_{X}(\cdot)$ and $\operatorname{PDF} f_{X}(\cdot)$ and the associated stress variables have the distribution with $\operatorname{PDF} f_{Y}(\cdot)$. The joint density function of (3) can be expressed as:

$$
L(\text { data }) \propto \prod_{i=1}^{n}\left(\prod_{j=1}^{s} f_{X}\left(x_{i j}\right)\right)\left[1-F_{X}\left(x_{i s}\right]^{k-s} f_{Y}\left(y_{i}\right)\right.
$$

Regarding the advantages of the likelihood function (4), one could observe that when $s=1$, it refers to the likelihood function for MSR with a conventional series system and in the case of $s=k$, it is the likelihood function for the parallel system. 


\section{Estimation of MSR with Common Location Parameters}

In this section, estimation is considered for MSR parameter $R_{s, k}$ when the strength and stress variables have the common Rayleigh location parameter $\alpha$, where $\alpha$ satisfies condition $0<\alpha<\min \left\{\min \left\{x_{i j}\right\}_{i=1, \ldots, n_{n}}^{j=1, \ldots,} y_{(1)}\right\}$. Two different approaches are proposed to establish point and interval estimates for MSR.

Let $X=\left\{X_{i 1}, X_{i 2}, \ldots, X_{i s}: i=1,2, \ldots, n\right\}$ be the strength variables of $\operatorname{RL}\left(\lambda_{1}, \alpha\right)$ and $Y=\left(Y_{1}, Y_{2}, \ldots, Y_{n}\right)$ be the associated stress variables of $\operatorname{RL}\left(\lambda_{2}, \alpha\right)$. Based on (1) and (2), the MSR parameter $R_{s, k}$ under this case can be written as:

$$
\begin{aligned}
R_{s, k} & =\sum_{i=s}^{k}\left(\begin{array}{c}
k \\
i
\end{array}\right) \int_{-\infty}^{\infty}\left[1-F\left(t ; \lambda_{1}, \alpha\right)\right]^{i}\left[F\left(t ; \lambda_{1}, \alpha\right)\right]^{k-i} d F\left(t ; \lambda_{2}, \alpha\right) \\
& =\sum_{i=s}^{k} \sum_{j=0}^{k-i}\left(\begin{array}{c}
k \\
i
\end{array}\right)\left(\begin{array}{c}
k-i \\
j
\end{array}\right) \frac{(-1)^{j} \lambda_{2}}{(i+j) \lambda_{1}+\lambda_{2}} .
\end{aligned}
$$

\subsection{Likelihood Based Inference for MSR}

In this subsection, maximum likelihood estimator (MLE) together with existence and uniqueness is established, and the associated approximate confidence interval (ACI) is also constructed based on the large sample theory and delta technique.

\subsubsection{Maximum Likelihood Estimation}

From (2) and (4) and based on the observed sample (3), the likelihood function of $\lambda_{1}, \lambda_{2}, \alpha$ can be expressed as:

$$
\begin{aligned}
L_{1}\left(\lambda_{1}, \lambda_{2}, \alpha\right) & \propto \prod_{i=1}^{n}\left(\prod_{j=1}^{s} f\left(x_{i j} ; \lambda_{1}, \alpha\right)\right)\left[1-F\left(x_{i s} ; \lambda_{1}, \alpha\right)\right]^{k-s} f\left(y_{i} ; \lambda_{2}, \alpha\right) \\
& \propto \lambda_{1}^{n s} \lambda_{2}^{n}\left(\prod_{i=1}^{n} \prod_{j=1}^{s}\left(x_{i j-\alpha}\right)\right)\left(\prod_{i=1}^{n}\left(y_{i}-\alpha\right)\right) \cdot \exp \left\{-\lambda_{2} \sum_{i=1}^{n}\left(y_{i}-\alpha\right)^{2}\right\} \\
& \cdot \exp \left\{-\lambda_{1}\left(\sum_{i=1}^{n} \sum_{j=1}^{s}\left(x_{i j}-\alpha\right)^{2}+(k-s) \sum_{i=1}^{n}\left(x_{i s}-\alpha\right)^{2}\right)\right\}
\end{aligned}
$$

and the associated log-likelihood function is given by:

$$
\begin{aligned}
& \ell_{1}\left(\lambda_{1}, \lambda_{2}, \alpha\right) \propto n s \ln \lambda_{1}+n \ln \lambda_{2}+\sum_{i=1}^{n} \sum_{j=1}^{s} \ln \left(x_{i j}-\alpha\right)+\sum_{i=1}^{n} \ln \left(y_{i}-\alpha\right) \\
& -\lambda_{1}\left(\sum_{i=1}^{n} \sum_{j=1}^{s}\left(x_{i j}-\alpha\right)^{2}+(k-s) \sum_{i=1}^{n}\left(x_{i s}-\alpha\right)^{2}\right)-\lambda_{2} \sum_{i=1}^{n}\left(y_{i}-\alpha\right)^{2} .
\end{aligned}
$$

Theorem 1. Suppose the strength and stress failure times follow Rayleigh distributions with parameters $\left(\lambda_{1}, \alpha\right)$ and $\left(\lambda_{2}, \alpha\right)$, respectively. For given $\alpha$, MLEs of $\lambda_{1}$ and $\lambda_{2}$ exist which can be obtained as:

$$
\begin{aligned}
& \dot{\lambda}_{1}=\frac{n s}{\sum_{i=1}^{n} \sum_{j=1}^{s}\left(x_{i j}-\alpha\right)^{2}+(k-s) \sum_{i=1}^{n}\left(x_{i s}-\alpha\right)^{2}} \\
& \dot{\lambda}_{2}=\frac{n}{\sum_{i=1}^{n}\left(y_{i}-\alpha\right)^{2}} .
\end{aligned}
$$

Proof. See Appendix A.

From (8), substituting $\dot{\lambda}_{1}=\lambda_{1}(\alpha)$ and $\dot{\lambda}_{2}=\lambda_{2}(\alpha)$ into (7), the profile log-likelihood function of $\alpha$ is obtained as: 


$$
\begin{aligned}
\ell_{11}(\alpha) & \propto-n s \ln \left[\sum_{i=1}^{n} \sum_{j=1}^{s}\left(x_{i j}-\alpha\right)^{2}+(k-s) \sum_{i=1}^{n}\left(x_{i s}-\alpha\right)^{2}\right]-n \ln \left[\sum_{i=1}^{n}\left(y_{i}-\alpha\right)^{2}\right] \\
& +\sum_{i=1}^{n} \sum_{j=1}^{s} \ln \left(x_{i j}-\alpha\right)+\sum_{i=1}^{n} \ln \left(y_{i}-\alpha\right) .
\end{aligned}
$$

Theorem 2. The MLE $\hat{\alpha}$ of $\alpha$ uniquely exists, which can be obtained from the following equation:

$$
\begin{aligned}
& \sum_{i=1}^{n} \sum_{j=1}^{s} \frac{1}{x_{i j}-\alpha}+\sum_{i=1}^{n} \frac{1}{y_{i}-\alpha}-\frac{2 n \sum_{i=1}^{n}\left(y_{i}-\alpha\right)}{\sum_{i=1}^{n}\left(y_{i}-\alpha\right)^{2}} \\
& -\frac{2 n s\left[\sum_{i=1}^{n} \sum_{j=1}^{s}\left(x_{i j}-\alpha\right)+(k-s) \sum_{i=1}^{n}\left(x_{i s}-\alpha\right)\right]}{\sum_{i=1}^{n} \sum_{j=1}^{s}\left(x_{i j}-\alpha\right)^{2}+(k-s) \sum_{i=1}^{n}\left(x_{i s}-\alpha\right)^{2}}=0 .
\end{aligned}
$$

Proof. See Appendix B.

Since the MLE $\hat{\alpha}$ does not have explicit form in the non-linear Equation (10), it can be obtained by using an iterative procedure such as the Newton-Raphson method. Then, the MLEs of $\lambda_{1}$ and $\lambda_{2}$ can be obtained from (8) respectively as:

$$
\begin{aligned}
& \hat{\lambda}_{1}=\frac{n s}{\sum_{i=1}^{n} \sum_{j=1}^{s}\left(x_{i j}-\hat{\alpha}\right)^{2}+(k-s) \sum_{i=1}^{n}\left(x_{i s}-\hat{\alpha}\right)^{2}}, \\
& \hat{\lambda}_{2}=\frac{n}{\sum_{i=1}^{n}\left(y_{i}-\hat{\alpha}\right)^{2}} .
\end{aligned}
$$

Therefore, the MLE of MSR parameter $R_{s, k}$ can be obtained from (5) as:

$$
\hat{R}_{s, k}=\sum_{i=s}^{k} \sum_{j=0}^{k-i}\left(\begin{array}{c}
k \\
i
\end{array}\right)\left(\begin{array}{c}
k-i \\
j
\end{array}\right) \frac{(-1)^{j} \hat{\lambda}_{2}}{(i+j) \hat{\lambda}_{1}+\hat{\lambda}_{2}} .
$$

\subsubsection{Approximate Confidence Interval}

Since it is difficult to pursue the exact distribution of the MLE for $R_{s, k}$, the exact confidence interval cannot be obtained either. In this subsection, an ACI for $R_{s, k}$ is constructed based on large sample theory and delta method.

The Fisher information matrix of parameter $\eta=\left(\lambda_{1}, \lambda_{2}, \alpha\right)$ is given by:

$$
I(\eta)=\left(\begin{array}{ccc}
-\frac{\partial^{2} \ell_{1}}{\partial \lambda_{1}^{2}} & -\frac{\partial^{2} \ell_{1}}{\partial \lambda_{1} \partial \lambda_{2}} & -\frac{\partial^{2} \ell_{1}}{\partial \lambda_{1} \partial \alpha} \\
-\frac{\partial^{2} \ell_{1}}{\partial \lambda_{1} \partial \lambda_{2}} & -\frac{\partial^{2} \ell_{1}}{\partial \lambda_{2}^{2}} & -\frac{\partial^{2} \ell_{1}}{\partial \lambda_{2} \partial \alpha} \\
-\frac{\partial^{2} \ell_{1}}{\partial \lambda_{1} \partial \alpha} & -\frac{\partial^{2} \ell_{1}}{\partial \lambda_{2} \partial \alpha} & -\frac{\partial^{2} \ell_{1}}{\partial \alpha^{2}}
\end{array}\right)
$$

where the second derivatives could be obtained directly of which the detailed expressions are omitted here for concision.

Theorem 3. When $n \rightarrow \infty, \sqrt{n} \hat{\eta}-\sqrt{n} \eta \stackrel{d}{\longrightarrow} N\left(0, n I^{-1}(\eta)\right)$, where $\stackrel{d}{\longrightarrow}$ means 'distributed as'.

Proof. Using the asymptotic properties of MLEs and multivariate central limit theorem, the result can be proven.

Based on Theorem 3, the following result for MSR is provided.

Theorem 4. Let $R_{s, k}$ be the MSR parameter defined in (5), when $n \rightarrow \infty$, one has:

$$
\sqrt{n} \hat{R}_{s, k}-\sqrt{n} R_{s, k} \stackrel{d}{\longrightarrow} N(0, n U(\eta)),
$$


where $U(\eta)=\left(\frac{\partial R_{s, k}}{\partial \eta}\right)^{T} I^{-1}(\eta)\left(\frac{\partial R_{s, k}}{\partial \eta}\right)$ and $\frac{\partial R_{s, k}}{\partial \eta}=\left(\frac{\partial R_{s, k}}{\partial \lambda_{1}}, \frac{\partial R_{s, k}}{\partial \lambda_{2}}, \frac{\partial R_{s, k}}{\partial \alpha}\right)^{T}$.

Proof. See Appendix C.

Replacing $\eta$ by its MLE $\hat{\eta}$, for arbitrary $0<\gamma<1$, an ACI of $R_{s, k}$ can be constructed from Theorem 4 as:

$$
\left(\hat{R}_{s, k}-z_{\gamma / 2} \sqrt{\widehat{\operatorname{Var}}\left(\hat{R}_{s, k}\right)}, \hat{R}_{s, k}+z_{\gamma / 2} \sqrt{\widehat{\operatorname{Var}}\left(\hat{R}_{s, k}\right)}\right),
$$

where $\widehat{\operatorname{Var}}\left(\hat{R}_{s, k}\right)={\widehat{\left(\frac{\partial R_{s, k}}{\partial \eta}\right)}}^{T} \widehat{\operatorname{Var}}(\hat{\eta}) \widehat{\left(\frac{\partial R_{s, k}}{\partial \eta}\right)}, \widehat{\operatorname{Var}}(\hat{\eta})=I^{-1}(\hat{\eta})$ and

$$
\widehat{\left(\frac{\partial R_{s, k}}{\partial \eta}\right)}=\left.\left(\frac{\partial R_{s, k}}{\partial \lambda_{1}}, \frac{\partial R_{s, k}}{\partial \lambda_{2}}, \frac{\partial R_{s, k}}{\partial \alpha}\right)^{T}\right|_{\eta=\hat{\eta}} .
$$

The ACI obtained by previous procedure may have a negative lower bound. In order to overcome this drawback, the logarithmic transformation and delta methods can be used to obtain the asymptotic normality distribution of $\ln \hat{R}_{s, k}$ as:

$$
\frac{\ln \hat{R}_{s, k}-\ln R_{s, k}}{\sqrt{\operatorname{Var}\left(\ln \hat{R}_{s, k}\right)}} \stackrel{d}{\rightarrow} N(0,1) .
$$

Therefore, a $100(1-\gamma) \%$ ACI of $R_{s, k}$ obtained via this manner can be constructed as:

$$
\left(\frac{\hat{R}_{s, k}}{\exp \left(z_{\gamma / 2} \sqrt{\widehat{\operatorname{Var}}\left(\ln \hat{R}_{s, k}\right)}\right)}, \hat{R}_{s, k} \exp \left(z_{\gamma / 2} \sqrt{\widehat{\operatorname{Var}}\left(\ln \hat{R}_{s, k}\right)}\right)\right)
$$

where $\widehat{\operatorname{Var}}\left(\ln \hat{R}_{s, k}\right)=\widehat{\operatorname{Var}}\left(\hat{R}_{s, k}\right) / \hat{R}_{s, k}^{2}$.

\subsection{Pivotal Based Inference for MSR}

A series of pivotal quantities are constructed based on the random samples of Rayleigh stress and strength in this subsection. Then pivotal quantities based estimators for MSR parameter $R_{s, k}$ are proposed correspondingly.

Theorem 5. Let $X=\left\{X_{i 1}, X_{i 2}, \ldots, X_{i s}: i=1,2, \ldots, n\right\}$ be the strength variable from $R L\left(\lambda_{1}, \alpha\right)$ and pivotal quantities be:

$$
P_{1}^{X}(\alpha)=2 \sum_{i=1}^{n} \sum_{j=1}^{s-1} \ln \left[\frac{(k-s)\left(X_{i s}-\alpha\right)^{2}+\sum_{r=1}^{s}\left(X_{i r}-\alpha\right)^{2}}{(k-j)\left(X_{i j}-\alpha\right)^{2}+\sum_{r=1}^{j}\left(X_{i r}-\alpha\right)^{2}}\right]
$$

and

$$
Q_{1}^{X}\left(\lambda_{1}, \alpha\right)=2 \lambda_{1} \sum_{i=1}^{n}\left\{(k-s)\left(X_{i s}-\alpha\right)^{2}+\sum_{r=1}^{s}\left(X_{i r}-\alpha\right)^{2}\right\} .
$$

Then $P_{1}^{X}(\lambda)$ and $Q_{1}^{X}\left(\lambda_{1}, \alpha\right)$ are statistically independent and follow chi-square distributions with $2 n(s-1)$ and 2 ns degrees of freedom, respectively.

Proof. See Appendix D. 
Theorem 6. Let $Y=\left(Y_{1}, Y_{2}, \ldots, Y_{n}\right)$ be the stress variable from $R L\left(\lambda_{2}, \alpha\right)$,

$$
P_{1}^{Y}(\alpha)=2 \sum_{j=1}^{n-1} \ln \left[\frac{\sum_{r=1}^{n}\left(Y_{(r)}-\alpha\right)^{2}}{(n-j)\left(Y_{(j)}-\alpha\right)^{2}+\sum_{r=1}^{j}\left(Y_{(r)}-\alpha\right)^{2}}\right]
$$

and

$$
Q_{1}^{Y}\left(\lambda_{2}, \alpha\right)=2 M_{n}=2 \lambda_{2} \sum_{r=1}^{n}\left(Y_{(r)}-\alpha\right)^{2},
$$

where $y_{(j)}$ is $j$ th order statistic of $Y$. Then $P_{1}^{Y}(\alpha)$ and $Q_{1}^{Y}\left(\lambda_{2}, \alpha\right)$ are statistically independent and have chi-square distributions with $2(n-1)$ and $2 n$ degrees of freedom, respectively.

Proof. See Appendix E.

In order to construct pivotal quantities, another useful lemma is also provided below.

Lemma 1. For arbitrary values $a$ and $b$ with $t<a<b$, function $K(t)=\left(\frac{b-t}{a-t}\right)^{2}$ increases in $t$.

Proof. The result is obvious and the proof is omitted.

Corollary 1. Pivotal quantities $P_{1}^{X}(\alpha)$ and $P_{1}^{Y}(\alpha)$ both increase in $\alpha$.

Proof. See Appendix F.

Based on Theorems 5 and 6, using independent property of $P_{1}^{X}(\alpha)$ and $P_{1}^{Y}(\alpha)$, pivotal quantity:

$$
\begin{aligned}
P_{1}(\alpha) & =P_{1}^{X}(\alpha)+P_{1}^{Y}(\alpha) \\
& =2 \sum_{i=1}^{n} \sum_{j=1}^{s-1} \ln \left[\frac{(k-s)\left(X_{i s}-\alpha\right)^{2}+\sum_{r=1}^{s}\left(X_{i r}-\alpha\right)^{2}}{(k-j)\left(X_{i j}-\alpha\right)^{2}+\sum_{r=1}^{j}\left(X_{i r}-\alpha\right)^{2}}\right] \\
& +2 \sum_{j=1}^{n-1} \ln \left[\frac{\sum_{r=1}^{n}\left(Y_{(r)}-\alpha\right)^{2}}{(n-j)\left(Y_{(j)}-\alpha\right)^{2}+\sum_{r=1}^{j}\left(Y_{(r)}-\alpha\right)^{2}}\right]
\end{aligned}
$$

follows the chi-square distribution with $2(n s-1)$ degrees of freedom. It is also deduced from Corollary 1 that $P_{1}(\alpha)$ increases in $\alpha$.

For a given $P_{1} \sim \chi_{2(n s-1)}^{2}$, equation $P_{1}(\alpha)=P_{1}$ has an unique solution in terms of $\alpha$ which is denoted by $h_{1}\left(P_{1} ; X, Y\right)$ and can be obtained by the bisection method. Moreover, from Theorem 5, one has:

$$
\lambda_{1}=\frac{Q_{1}^{X}}{H_{1}^{X}[\alpha]} \quad \text { and } \quad H_{1}^{X}[\alpha]=2 \sum_{i=1}^{n}\left\{(k-s)\left(x_{i s}-\alpha\right)^{2}+\sum_{r=1}^{s}\left(x_{i r}-\alpha\right)^{2}\right\}
$$

Following the substitution method of Weerahandi [20], a generalized pivotal quantity, namely $S_{1}^{X}$, can be constructed by substituting $h_{1}\left(P_{1} ; X, Y\right)$ for $\alpha$ in the expression of $\lambda_{1}$ as follows:

$$
\begin{aligned}
S_{1}^{X} & =\frac{Q_{1}^{X}}{2 \sum_{i=1}^{n}\left\{(k-s)\left(x_{i s}-h_{1}\left(P_{1} ; x, y\right)\right)^{2}+\sum_{r=1}^{s}\left(x_{i r}-h_{1}\left(P_{1} ; x, y\right)\right)^{2}\right\}} \\
& =\frac{\sum_{i=1}^{n}\left\{(k-s)\left(x_{i s}-h_{1}\left(P_{1} ; X, Y\right)\right)^{2}+\sum_{r=1}^{s}\left(x_{i r}-h_{1}\left(P_{1} ; X, Y\right)\right)^{2}\right\}}{\sum_{i=1}^{n}\left\{(k-s)\left(x_{i s}-h_{1}\left(P_{1} ; x, y\right)\right)^{2}+\sum_{r=1}^{s}\left(x_{i r}-h_{1}\left(P_{1} ; x, y\right)\right)^{2}\right\}} \cdot \lambda_{1} \\
& =\frac{Q_{1}^{X}}{H_{1}^{X}\left[h_{1}\left(P_{1} ; x, y\right)\right]},
\end{aligned}
$$


where $(x, y)$ denotes the observation of sample $(X, Y)$. It is noted that the distribution of $S_{1}^{X}$ is free of any unknown parameters in its first expression, and that $S_{1}$ reduces to $\lambda_{1}$ when $(X, Y)=(x, y)$. Therefore, $S_{1}^{X}$ is a generalized pivotal quantity for parameter $\lambda_{1}$. Similarly, from Theorem 6, a generalized pivotal quantity for parameter $\lambda_{2}$ could be constructed as:

$$
S_{1}^{Y}=\frac{Q_{1}^{Y}}{H_{1}^{Y}\left[h_{1}\left(P_{1} ; x, y\right)\right]} \quad \text { with } \quad H_{1}^{Y}[\alpha]=2 \sum_{r=1}^{n}\left[y_{(r)}-\alpha\right]^{2} \quad \text { and } \quad Q_{1}^{Y} \sim \chi_{2 n}^{2} .
$$

Therefore, a generalized pivotal quantity for $R_{s, k}$ can be constructed as:

$$
W_{1}=\sum_{i=s}^{k} \sum_{j=0}^{k-i}\left(\begin{array}{c}
k \\
i
\end{array}\right)\left(\begin{array}{c}
k-i \\
j
\end{array}\right) \frac{(-1)^{j}}{1+(i+j) \frac{Q_{1}^{X}}{Q_{1}^{Y}} \frac{H_{1}^{Y}\left[h_{1}\left(P_{1} ; x, y\right)\right]}{H_{1}^{X}\left[h_{1}\left(P_{1} ; x, y\right)\right]}} .
$$

Correspondingly, a procedure to estimate $R_{s, k}$ is provided as Algorithm 1 .

Algorithm 1: Pivotal based estimation for $R_{s, k}$ with common parameter.

Step 1 Generate a realization $p_{1}$ of $P_{1}$ from chi-squared distribution with $2(n s-1)$ degrees of freedom. Then an observation $h_{1}$ of $h_{1}\left(P_{1} ; X, Y\right)$ can be obtained from the equation $P_{1}(\lambda)=p_{1}$.

Step 2 Generate random data for $Q_{1}^{X}$ and $Q_{1}^{Y}$ from chi-squared distribution with $2 n s$ and $2 n$ degrees of freedom, respectively. Then compute $W_{1}$.

Step 3 Repeat Step 1 and $2 N$ times, one can obtain $N$ values of $W_{1}$ as $W_{1}^{(1)}, W_{1}^{(2)}, \ldots, W_{1}^{(N)}$.

Step 4 Two types of point estimators are proposed here. One natural generalized point estimator for $R_{s, k}$ is given by:

$$
\dot{R}_{s, k}=\frac{1}{N} \sum_{j=1}^{N} W_{1}^{(j)} .
$$

Moreover, an alternative point estimator utilizing Fisher $Z$ transformation is given as:

$$
\hat{R}_{s, k}^{F}=\frac{\exp \left\{\frac{1}{N} \sum_{j=1}^{N} \ln \left[\frac{1+W_{1}^{(j)}}{1-W_{1}^{(j)}}\right]\right\}-1}{\exp \left\{\frac{1}{N} \sum_{j=1}^{N} \ln \left[\frac{1+W_{1}^{(j)}}{1-W_{1}^{(j)}}\right]\right\}+1} .
$$

Step 5 Arrange all estimates of $W_{1}$ in ascending order as $W_{1}^{[1]}, W_{1}^{[2]}, \ldots, W_{1}^{[N]}$. For arbitrary $0<\gamma<1$, a series of $100(1-\gamma)$ confidence intervals of $R_{s, k}$ can be constructed as $\left(W_{1}^{[j]}, W_{1}^{[j+N-[N \gamma+1]]}\right)$, $j=1,2, \ldots,[N \gamma]$, where $[t]$ denotes the greatest integer less than or equal to $t$. Therefore, a $100(1-\gamma) \%$ generalized confidence interval (GCI) of $R_{s, k}$ can be constructed as the $j^{*}$ th one satisfying:

$$
W_{1}^{\left[j^{*}+N-[N \gamma+1]\right]}-W_{1}^{\left[j^{*}\right]}=\min _{j=1}^{[N \gamma]}\left(W_{1}^{[j+N-[N \gamma+1]]}-W_{1}^{[j]}\right)
$$

Besides previous proposed generalized estimates, some applications are provided as complementary.

Remark 1. Using pivotal quantity $P_{1}(\alpha)$, for arbitrary $0<\gamma<1$, a $100(1-\gamma) \%$ exact confidence interval for $\alpha$ is given by:

$$
\left(h_{1}\left(\chi_{2(n s-1)}^{1-\gamma / 2} ; X, Y\right), h_{1}\left(\chi_{2(n s-1)}^{\gamma / 2} ; X, Y\right)\right)
$$

where $\chi_{k}^{\gamma}$ denotes the right-tail $100 \gamma \%$ quantile of the chi-square distribution with $k$ degrees of freedom. 


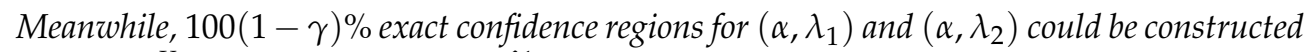
from $\left(P_{1}(\alpha), Q_{1}^{X}\left(\lambda_{1}, \alpha\right)\right)$, and $\left(P_{1}(\alpha), Q_{1}^{Y}\left(\lambda_{2}, \alpha\right)\right)$ as follows:

$$
\left\{\left(\alpha, \lambda_{1}\right): h_{1}\left(\chi_{2(n s-1)}^{\frac{1-\sqrt{1-\gamma}}{2}} ; X, Y\right)<\alpha<h_{1}\left(\chi_{2(n s-1)}^{\frac{1+\sqrt{1-\gamma}}{2}} ; X, Y\right), \frac{\chi_{2 n s}^{\frac{1-\sqrt{1-\gamma}}{2}}}{H_{1}^{X}[\alpha]}<\lambda_{1}<\frac{\chi_{2 n s}^{\frac{1+\sqrt{1-\gamma}}{2}}}{H_{1}^{X}[\alpha]}\right\}
$$

and

$$
\left\{\left(\alpha, \lambda_{2}\right): h_{1}\left(\chi_{2(n s-1)}^{\frac{1-\sqrt{1-\gamma}}{2}} ; X, Y\right)<\alpha<h_{1}\left(\chi_{2(n s-1)}^{\frac{1+\sqrt{1-\gamma}}{2}} ; X, Y\right), \frac{\chi_{2 n}^{\frac{1-\sqrt{1-\gamma}}{2}}}{H_{1}^{Y}[\alpha]}<\lambda_{2}<\frac{\chi_{2 n}^{\frac{1+\sqrt{1-\gamma}}{2}}}{H_{1}^{Y}[\alpha]}\right\}
$$

respectively.

Remark 2. Consider null hypothesis $H_{0}$ and alternative hypothesis $H_{1}$ listed as follows:

$$
\begin{aligned}
& \text { (a) } H_{0}: \alpha \leq \alpha_{0} \leftrightarrow H_{1}: \alpha>\alpha_{0}, \\
& \text { (b) } H_{0}: \alpha \geq \alpha_{0} \leftrightarrow H_{1}: \alpha<\alpha_{0}, \\
& \text { (c) } H_{0}: \alpha=\alpha_{0} \leftrightarrow H_{1}: \alpha \neq \alpha_{0} .
\end{aligned}
$$

For arbitrary $0<\gamma<1$, the decision rule to reject null hypothesis $H_{0}$ in $(a),(b)$, (c) can be expressed as:

$$
\begin{aligned}
& (a)^{\prime}\left\{P_{1}\left(\alpha_{0}\right) \geq \chi_{2(n s-1)}^{\gamma}\right\}, \quad(b)^{\prime}\left\{P_{1}\left(\alpha_{0}\right) \leq \chi_{2(n s-1)}^{\gamma}\right\}, \\
& (c)^{\prime}\left\{P_{1}\left(\alpha_{0}\right) \leq \chi_{2(n s-1)}^{\gamma / 2} \text { or } P_{1}\left(\alpha_{0}\right) \geq \chi_{2(n s-1)}^{1-\gamma / 2}\right\},
\end{aligned}
$$

respectively.

\section{Estimation for MSR with Unequal Parameters}

In this section, inference for MSR parameter $R_{s, k}$ is studied under unequal location parameters, i.e., strength variable $X=\left\{X_{i 1}, X_{i 2}, \ldots, X_{i s}: i=1,2, \ldots, n\right\}$ comes from $\operatorname{RL}\left(\lambda_{1}, \alpha_{1}\right)$ with $0<\alpha_{1}<\min \left\{x_{i 1}\right\}_{i=1}^{n}$, and the associated stress variable $Y=\left\{Y_{1}, Y_{2}, \ldots, Y_{s}\right\}$ follows $\operatorname{RL}\left(\lambda_{2}, \alpha_{2}\right)$ with $0<\alpha_{2}<y_{(1)}$.

In this case, denote $\alpha_{0}=\max \left\{\alpha_{1}, \alpha_{2}\right\}$ with $\alpha_{1} \neq \alpha_{2}$ and the MSR parameter $R_{s, k}$ is given by:

$$
\begin{aligned}
& R_{s, k}=\sum_{i=s}^{k}\left(\begin{array}{c}
k \\
i
\end{array}\right) \int_{-\infty}^{\infty}\left[1-F\left(t ; \lambda_{1}, \alpha_{1}\right)\right]^{i}\left[F\left(t ; \lambda_{1}, \alpha_{1}\right)\right]^{k-i} d F\left(t ; \lambda_{2}, \alpha_{2}\right) \\
& =\sum_{i=s}^{k} \sum_{j=0}^{k-i}\left(\begin{array}{c}
k \\
i
\end{array}\right)\left(\begin{array}{c}
k-i \\
j
\end{array}\right)(-1)^{j} \int_{1-e^{-\lambda_{2}\left(\alpha_{0}-\alpha_{2}\right)^{2}}}^{1} e^{-\lambda_{1}(i+j)\left(\alpha_{2}-\alpha_{1}+\sqrt{\frac{-\ln (1-u)}{\lambda_{2}}}\right)^{2}} d u
\end{aligned}
$$

\subsection{Likelihood Based Inference for $R_{s, k}$}

From (2) and (4), the likelihood function of $\lambda_{1}, \alpha_{1}$ and $\lambda_{2}, \alpha_{2}$ can be expressed as:

$$
\begin{aligned}
L_{2}\left(\lambda_{1}, \alpha_{1}, \lambda_{2}, \alpha_{2}\right) & \propto \prod_{i=1}^{n}\left(\prod_{j=1}^{s} f\left(x_{i j} ; \lambda_{1}, \alpha_{1}\right)\right)\left[1-F\left(x_{i s} ; \lambda_{1}, \alpha_{1}\right)\right]^{k-s} f\left(y_{i} ; \lambda_{2}, \alpha_{2}\right) \\
& \propto \lambda_{1}^{n s} \lambda_{2}^{n}\left(\prod_{i=1}^{n} \prod_{j=1}^{s}\left(x_{i j}-\alpha_{1}\right) \prod_{i=1}^{n}\left(y_{i}-\alpha_{2}\right)\right) \exp \left\{-\lambda_{2} \sum_{i=1}^{n}\left(y_{i}-\alpha_{2}\right)^{2}\right\} \\
& \cdot \exp \left\{-\lambda_{1} \sum_{i=1}^{n} \sum_{j=1}^{s}\left(x_{i j}-\alpha_{1}\right)^{2}-\lambda_{1}(k-s) \sum_{i=1}^{n}\left(x_{i s}-\alpha_{1}\right)^{2}\right\}
\end{aligned}
$$


and the associated log-likelihood function can be written as:

$$
\begin{aligned}
& \ell_{2}\left(\lambda_{1}, \alpha_{1}, \lambda_{2}, \alpha_{2}\right) \propto n s \ln \lambda_{1}+n \ln \lambda_{2}+\sum_{i=1}^{n} \sum_{j=1}^{s} \ln \left(x_{i j}-\alpha_{1}\right)+\sum_{i=1}^{n} \ln \left(y_{i}-\alpha_{2}\right) \\
& -\lambda_{1}\left\{\sum_{i=1}^{n} \sum_{j=1}^{s}\left(x_{i j}-\alpha_{1}\right)^{2}+(k-s) \sum_{i=1}^{n}\left(x_{i s}-\alpha_{1}\right)^{2}\right\}-\lambda_{2} \sum_{i=1}^{n}\left(y_{i}-\alpha_{2}\right)^{2} .
\end{aligned}
$$

Following similar processes to prove Theorems 1 and 2, the following results can be proved.

Theorem 7. Let the latent strength and stress failure times follow Rayleigh distributions with parameters $\left(\lambda_{1}, \alpha_{1}\right)$ and $\left(\lambda_{2}, \alpha_{2}\right)$, respectively. Then MLEs $\check{\lambda}_{1}, \check{\lambda}_{2}, \check{\alpha}_{1}, \check{\alpha}_{2}$ of $\lambda_{1}, \lambda_{2}, \alpha_{1}, \alpha_{2}$ uniquely exist and are given by:

$$
\begin{aligned}
& \check{\lambda}_{1}=\frac{n s}{\sum_{i=1}^{n} \sum_{j=1}^{s}\left(x_{i j}-\check{\alpha}_{1}\right)^{2}+(k-s) \sum_{i=1}^{n}\left(x_{i s}-\check{\alpha}_{1}\right)^{2}} \\
& \check{\lambda}_{2}=\frac{n}{\sum_{i=1}^{n}\left(y_{i}-\check{\alpha}_{2}\right)^{2}},
\end{aligned}
$$

with $\check{\alpha}_{1}$ and $\check{\alpha}_{2}$ being the solutions of the following equations:

$$
\begin{aligned}
& \sum_{i=1}^{n} \sum_{j=1}^{s} \frac{1}{x_{i j}-\alpha_{1}}-\frac{2 n s\left[\sum_{i=1}^{n} \sum_{j=1}^{s}\left(x_{i j}-\alpha_{1}\right)+(k-s) \sum_{i=1}^{n}\left(x_{i s}-\alpha_{1}\right)\right]}{\sum_{i=1}^{n} \sum_{j=1}^{s}\left(x_{i j}-\alpha_{1}\right)^{2}+(k-s) \sum_{i=1}^{n}\left(x_{i s}-\alpha_{1}\right)^{2}}=0, \\
& \sum_{i=1}^{n} \frac{1}{y_{i}-\alpha_{2}}-\frac{2 n \sum_{i=1}^{n}\left(y_{i}-\alpha_{2}\right)}{\sum_{i=1}^{n}\left(y_{i}-\alpha_{2}\right)^{2}}=0 .
\end{aligned}
$$

Using the invariant property of maximum likelihood estiamtion, the MLE of MSR parameter $R_{s, k}$ is given by:

$$
\check{R}_{s, k}=\sum_{i=s}^{k} \sum_{j=0}^{k-i}\left(\begin{array}{c}
k \\
i
\end{array}\right)\left(\begin{array}{c}
k-i \\
j
\end{array}\right)(-1)^{j} \int_{1-e^{-\check{\lambda}_{2}\left(\check{x}_{0}-\check{\alpha}_{2}\right)^{2}}}^{1} e^{-\check{\lambda}_{1}(i+j)\left(\check{\alpha}_{2}-\check{x}_{1}+\sqrt{\frac{-\ln (1-u)}{\grave{\lambda}_{2}}}\right)^{2}} d u
$$

with $\check{\alpha}_{0}=\max \left\{\check{\alpha}_{1}, \check{\alpha}_{2}\right\}$.

The observed Fisher information matrix of parameter $v=\left(\lambda_{1}, \alpha_{1}, \lambda_{2}, \alpha_{2}\right)$ is given by:

$$
J(v)=\left(\begin{array}{cccc}
-\frac{\partial^{2} \ell_{2}}{\partial \lambda_{1}^{2}} & -\frac{\partial^{2} \ell_{1}}{\partial \lambda_{1} \partial \alpha_{1}} & 0 & 0 \\
-\frac{\partial^{2} \ell_{2}}{\partial \lambda_{1} \partial \alpha_{1}} & -\frac{\partial^{2} \ell_{1}}{\partial \alpha_{1}^{2}} & 0 & 0 \\
0 & 0 & -\frac{\partial^{2} \ell_{2}}{\partial \lambda_{2}^{2}} & -\frac{\partial^{2} \ell_{2}}{\partial \lambda_{2} \partial \alpha_{2}} \\
0 & 0 & -\frac{\partial^{2} \ell_{2}}{\partial \lambda_{2} \partial \alpha_{2}} & -\frac{\partial^{2} \ell_{2}}{\partial \alpha_{2}^{2}}
\end{array}\right)
$$

where the second derivatives could be obtained directly of which the detailed expressions are omitted here for concision.

Therefore, following a similar procedure to develop Theorem 4 and replacing $v$ by its MLE $\hat{v}$, for arbitrary $0<\gamma<1$, an ACI of $R_{s, k}$ can be constructed as:

$$
\left(\check{R}_{s, k}-z_{\gamma / 2} \sqrt{\widetilde{\operatorname{Var}}\left(\check{R}_{s, k}\right)}, \check{R}_{s, k}+z_{\gamma / 2} \sqrt{\widetilde{\operatorname{Var}}\left(\check{R}_{s, k}\right)}\right),
$$


where

$$
\widetilde{\operatorname{Var}}\left(\check{R}_{s, k}\right)=\left(\frac{\partial R_{s, k}}{\partial v}\right)^{T} \widetilde{\operatorname{Var}}(\check{\eta})\left(\frac{\partial R_{s, k}}{\partial v}\right), \quad \widetilde{\operatorname{Var}}(\check{v})=J^{-1}(\check{v}),
$$

and

$$
\frac{\partial R_{s, k}}{\partial v}=\left.\left(\frac{\partial R_{s, k}}{\partial \lambda_{1}}, \frac{\partial R_{s, k}}{\partial \alpha_{1}}, \frac{\partial R_{s, k}}{\partial \lambda_{2}}, \frac{\partial R_{s, k}}{\partial \alpha_{2}}\right)^{T}\right|_{v=\check{v}}
$$

Moreover, an alternative $100(1-\gamma) \% \mathrm{ACI}$ of $R_{s, k}$ is given by:

$$
\left(\frac{\check{R}_{s, k}}{\exp \left(z_{\gamma / 2} \sqrt{\widetilde{\operatorname{Var}}\left(\ln \check{R}_{s, k}\right)}\right)}, \check{R}_{s, k} \exp \left(z_{\gamma / 2} \sqrt{\widetilde{\operatorname{Var}}\left(\ln \check{R}_{s, k}\right)}\right)\right),
$$

where $\widetilde{\operatorname{Var}}\left(\ln \check{R}_{s, k}\right)=\widetilde{\operatorname{Var}}\left(\check{R}_{s, k}\right) / \check{R}_{s, k}^{2}$.

4.2. Pivotal Based Inference for $R_{s, k}$

Following Theorems 5 and 6, one directly has:

Theorem 8. Let $X=\left\{X_{i 1}, X_{i 2}, \ldots, X_{i s}: i=1,2, \ldots, n\right\}$ and $Y=\left\{Y_{1}, X_{2}, \ldots, Y_{n}\right\}$ be independent strength and stress variables from $R L\left(\lambda_{1}, \alpha_{1}\right)$ and $R L\left(\alpha_{2}, \alpha_{2}\right)$, respectively. Denote pivotal quantities:

$$
\begin{aligned}
& P_{2}^{X}\left(\alpha_{1}\right)=2 \sum_{i=1}^{n} \sum_{j=1}^{s-1} \ln \left[\frac{(k-s)\left(X_{i s}-\alpha_{1}\right)^{2}+\sum_{r=1}^{s}\left(X_{i r}-\alpha_{1}\right)^{2}}{(k-j)\left(X_{i j}-\alpha_{1}\right)^{2}+\sum_{r=1}^{j}\left(X_{i r}-\alpha_{1}\right)^{2}}\right], \\
& Q_{2}^{X}\left(\lambda_{1}, \alpha_{1}\right)=2 \lambda_{1} \sum_{i=1}^{n}\left\{(k-s)\left(X_{i s}-\alpha_{1}\right)^{2}+\sum_{r=1}^{s}\left(X_{i r}-\alpha_{1}\right)^{2}\right\}
\end{aligned}
$$

and

$$
\begin{aligned}
& P_{2}^{Y}\left(\alpha_{2}\right)=2 \sum_{j=1}^{n-1} \ln \left[\frac{\sum_{r=1}^{n}\left(Y_{(r)}-\alpha_{2}\right)^{2}}{(n-j)\left(Y_{(j)}-\alpha_{2}\right)^{2}+\sum_{r=1}^{j}\left(Y_{(r)}-\alpha_{2}\right)^{2}}\right], \\
& Q_{2}^{Y}\left(\lambda_{2}, \alpha_{2}\right)=2 \lambda_{2} \sum_{r=1}^{n}\left(Y_{(r)}-\alpha_{2}\right)^{2} .
\end{aligned}
$$

Then,

- $\quad P_{2}^{X}\left(\alpha_{1}\right) \sim \chi_{2 n(s-1)}^{2}, Q_{2}^{X}\left(\lambda_{1}, \alpha_{1}\right) \sim \chi_{2 n s}^{2}$ are statistically independent;

- $\quad P_{2}^{Y}\left(\alpha_{2}\right) \sim \chi_{2(n-1)}^{2}, Q_{2}^{Y}\left(\lambda_{2}, \alpha_{2}\right) \sim \chi_{2 n}^{2}$ are statistically independent.

Similar to the process in Section 3, for given $P_{2}^{X} \sim \chi_{2 n(s-1)}^{2}$ and $P_{2}^{Y} \sim \chi_{2(n-1)}^{2}$, denote $h_{2}\left(P_{2}^{X} ; X\right)$ and $h_{2}\left(P_{2}^{Y} ; Y\right)$ as the solutions of equations $P_{2}^{X}(\lambda)=P_{2}^{X}$ and $P_{2}^{Y}(\lambda)=P_{2}^{Y}$, respectively. Using the substitution method of Weerahandi [20], generalized pivotal quantities for $\lambda_{1}$ and $\lambda_{2}$ can be constructed respectively as:

$$
S_{2}^{X}=\frac{Q_{2}^{X}}{H_{2}^{X}\left[h_{2}\left(P_{2}^{X} ; x\right)\right]}
$$


with $Q_{2}^{X} \sim \chi_{2 n s}^{2}$ and

$$
H_{2}^{X}\left[\alpha_{1}\right]=2 \sum_{i=1}^{n}\left\{(k-s)\left(x_{i s}-\alpha_{1}\right)^{2}+\sum_{r=1}^{s}\left(x_{i r}-\alpha_{1}\right)^{2}\right\},
$$

whereas,

$$
S_{2}^{Y}=\frac{Q_{2}^{Y}}{H_{2}^{Y}\left[h_{2}\left(P_{2}^{Y} ; y\right)\right]} \quad \text { with } \quad Q_{2}^{X} \sim \chi_{2 n}^{2}, H_{2}^{Y}\left[\alpha_{2}\right]=2 \sum_{r=1}^{n}\left(Y_{(r)}-\alpha_{2}\right)^{2} .
$$

Therefore, denote $h_{3}(x, y)=\max \left\{h_{2}\left(P_{2}^{X} ; x\right), h_{2}\left(P_{2}^{Y} ; y\right)\right\}$, a generalized pivotal quantity for $R_{s, k}$ can be expressed as:

$$
\begin{aligned}
W_{2} & =\sum_{i=s}^{k} \sum_{j=0}^{k-i}\left(\begin{array}{c}
k \\
i
\end{array}\right)\left(\begin{array}{c}
k-i \\
j
\end{array}\right)(-1)^{j} \int_{1-\exp \left\{-S_{2}^{Y}\left(h_{3}(x, y)-h_{2}\left(P_{2}^{Y} ; y\right)\right)^{2}\right\}}^{1} \\
& \exp \left\{-S_{2}^{X}(i+j)\left(h_{2}\left(P_{2}^{Y} ; y\right)-h_{2}\left(P_{2}^{X} ; x\right)+\sqrt{\frac{-\ln (1-u)}{S_{2}^{Y}}}\right)^{2}\right\} d u .
\end{aligned}
$$

Furthermore, the following Algorithm 2 is provided to obtain the aformentioned generalized estimates of $R_{s, k}$ under unequal parameters.

Algorithm 2: Pivotal based estimation for $R_{s, k}$ with unequal parameters

Step 1 Generate a realization $p_{21}$ of $P_{2}^{X}$ from the chi-squared distribution with $2 n(s-1)$ degrees of freedom. Then an observation $h_{21}$ of $h_{2}\left(P_{2}^{X} ; X\right)$ can be obtained from the equation $P_{2}^{X}\left(\lambda_{1}\right)=p_{21}$. Similarly, generate a realization $p_{22}$ of $P_{2}^{Y}$ from chi-squared distribution with $2(n-1)$ degrees of freedom, and an observation $h_{22}$ of $h_{2}\left(P_{2}^{Y} ; Y\right)$ is obtained from the equation $P_{2}^{Y}\left(\lambda_{2}\right)=p_{22}$.

Step 2 Generate random data for $Q_{2}^{X}$ and $Q_{2}^{Y}$ from the chi-squared distributions with $2 n s$ and $2 n$ degrees of freedom, respectively. Then compute $W_{2}$.

Step 3 Repeat Step 1 and $2 N$ times, one can obtain $N$ values of $W_{2}$ as $W_{2}^{(1)}, W_{2}^{(2)}, \ldots, W_{2}^{(N)}$.

Step 4 A natural generalized estimator $\grave{R}_{s, k}$ and a Fisher $Z$ transformation based estimator $\grave{R}_{s, k}^{F}$ for $R_{s, k}$ can be constructed as:

$$
\grave{R}_{s, k}=\frac{1}{N} \sum_{j=1}^{N} W_{2}^{(j)} \quad \text { and } \quad \grave{R}_{s, k}^{F}=\frac{\exp \left\{\frac{1}{N} \sum_{j=1}^{N} \ln \left[\frac{1+W_{2}^{(j)}}{1-W_{2}^{(j)}}\right]\right\}-1}{\exp \left\{\frac{1}{N} \sum_{j=1}^{N} \ln \left[\frac{1+W_{2}^{(j)}}{1-W_{2}^{(j)}}\right]\right\}+1} .
$$

Step 5 Arrange all estimates of $W_{2}$ in ascending order as $W_{2}^{[1]}, W_{2}^{[2]}, \ldots, W_{2}^{[N]}$. For arbitrary $0<\gamma<1$, a series of $100(1-\gamma)$ confidence intervals of $R_{s, k}$ can be constructed as $\left(W_{2}^{[j]}, W_{2}^{[j+N-[N \gamma+1]]}\right), j=1,2, \ldots,[N \gamma]$. Therefore, a $100(1-\gamma) \%$ GCI of $R_{s, k}$ can be obtained as the $j^{*}$ th one satisfying:

$$
W_{2}^{\left[j^{*}+N-[N \gamma+1]\right]}-W_{2}^{\left[j^{*}\right]}=\min _{j=1}^{[N \gamma]}\left(W_{2}^{[j+N-[N \gamma+1]]}-W_{2}^{[j]}\right) .
$$

Similarly, some applications are also presented below. 
Remark 3. For arbitrary $0<\gamma<1, a 100(1-\gamma) \%$ exact confidence interval for $\alpha_{1}$ and $\alpha_{2}$ is given by:

$$
\left(h_{2}\left(\chi_{2 n(n-1)}^{1-\gamma / 2} ; X\right), h_{2}\left(\chi_{2 n(s-1)}^{\gamma / 2} ; X\right)\right) \text { and }\left(h_{2}\left(\chi_{2(n-1)}^{1-\gamma / 2} ; Y\right), h_{2}\left(\chi_{2(n-1)}^{\gamma / 2} ; Y\right)\right),
$$

respectively. Furthermore, exact confidence regions for $\left(\lambda_{1}, \alpha_{1}\right)$ and $\left(\lambda_{2}, \alpha_{2}\right)$ are constructed as:

$$
\left\{\left(\alpha_{1}, \lambda_{1}\right): h_{2}\left(\chi_{2 n(s-1)}^{\frac{1-\sqrt{1-\gamma}}{2}} ; X\right)<\alpha_{1}<h_{2}\left(\chi_{2 n(s-1)}^{\frac{1+\sqrt{1-\gamma}}{2}} ; X\right), \frac{\chi_{2 n s}^{\frac{1-\sqrt{1-\gamma}}{2}}}{H_{2}^{X}\left[\alpha_{1}\right]}<\lambda_{1}<\frac{\chi_{2 n s}^{\frac{1+\sqrt{1-\gamma}}{2}}}{H_{2}^{X}\left[\alpha_{1}\right]}\right\}
$$

and

$$
\left\{\left(\alpha_{2}, \lambda_{2}\right): h_{2}\left(\chi_{2(n-1)}^{\frac{1-\sqrt{1-\gamma}}{2}} ; Y\right)<\alpha_{2}<h_{2}\left(\chi_{2(n-1)}^{\frac{1+\sqrt{1-\gamma}}{2}} ; Y\right), \frac{\chi_{2 n}^{\frac{1-\sqrt{1-\gamma}}{2}}}{H_{2}^{Y}\left[\alpha_{2}\right]}<\lambda_{2}<\frac{\chi_{2 n}^{\frac{1+\sqrt{1-\gamma}}{2}}}{H_{2}^{Y}\left[\alpha_{2}\right]}\right\},
$$

respectively.

Remark 4. For $i=1,2$, consider the following null hypothesis $H_{0}$ and alternative hypothesis $H_{1}$ :

$$
\begin{aligned}
& \text { (d) } H_{0}: \alpha_{i} \leq \alpha_{i 0} \leftrightarrow H_{1}: \alpha_{i}>\alpha_{i 0}, \\
& \text { (e) } H_{0}: \alpha_{i} \geq \alpha_{i 0} \leftrightarrow H_{1}: \alpha_{i}<\alpha_{i 0}, \\
& \text { (f) } H_{0}: \alpha_{i}=\alpha_{i 0} \leftrightarrow H_{1}: \alpha_{i} \neq \alpha_{i 0} .
\end{aligned}
$$

Therefore, under significance level $0<\gamma<1$, the decision rule to reject null hypothesis $H_{0}$ in $(d),(e),(f)$ for $\alpha_{1}$ and $\alpha_{2}$ can be expressed as:

$$
\begin{aligned}
& (d)^{\prime}\left\{P_{2}^{X}\left(\alpha_{10}\right) \geq \chi_{2 n(s-1)}^{\gamma}\right\}, \\
& (e)^{\prime}\left\{P_{2}^{X}\left(\alpha_{10}\right) \leq \chi_{2 n(s-1)}^{\gamma}\right\}, \\
& (f)^{\prime}\left\{P_{2}^{X}\left(\alpha_{10}\right) \leq \chi_{2 n(s-1)}^{\gamma / 2}, \text { or } P_{2}^{X}\left(\alpha_{10}\right) \geq \chi_{2 n(s-1)}^{1-\gamma / 2}\right\},
\end{aligned}
$$

and

$$
\begin{aligned}
& (d)^{\prime \prime}\left\{P_{2}^{Y}\left(\alpha_{20}\right) \geq \chi_{2(n-1)}^{\gamma}\right\}, \\
& (e)^{\prime \prime}\left\{P_{2}^{Y}\left(\alpha_{20}\right) \leq \chi_{2(n-1)}^{\gamma}\right\}, \\
& (f)^{\prime \prime}\left\{P_{2}^{Y}\left(\alpha_{20}\right) \leq \chi_{2(n-1)}^{\gamma / 2}, \text { or } P_{2}^{X}\left(\alpha_{20}\right) \geq \chi_{2(n-1)}^{1-\gamma / 2}\right\}
\end{aligned}
$$

respectively.

Remark 5. It is worth noting that for both common and unequal parameter cases, for computational purposes, the value of $s$ from the s-out-of- $k$ system must be at least 2, else the previous proposed pivotal quantities $P_{i}^{X}$ and $Q_{i}^{X}, i=1,2$ cannot be constructed with $s=1$. Under this situation, the strength variables $X_{11}, X_{21}, \ldots, X_{n 1}$ can be viewed as an i.i.d. sample of size $n$ from lifetime distribution with $\operatorname{CDF} F(t ; \alpha, \lambda)=1-e^{-k \lambda(t-\alpha)^{2}}$. As an alternative approach, one could construct the following pivotal quantities:

$$
P_{i}^{X}\left(\alpha_{(\cdot)}\right)=2 \sum_{j=1}^{n-1} \ln \left[\frac{\sum_{r=1}^{n}\left(X_{(r 1)}-\alpha(\cdot)\right)^{2}}{(n-j)\left(X_{(j 1)}-\alpha(\cdot)\right)^{2}+\sum_{r=1}^{j}\left(X_{(r 1)}-\alpha\right)^{2}}\right]
$$


and

$$
Q_{i}^{X}\left(\lambda_{1}, \alpha_{(\cdot)}\right)=2 \lambda_{1} \sum_{r=1}^{n}\left(X_{(r 1)}-\alpha(\cdot)\right)^{2}
$$

which have the chi-square distributions with $2(n-1)$ and $2 n$ degrees of freedom respectively, where $\alpha_{(\cdot)}=\alpha$ or $\alpha_{1}$ under common and unequal parameter cases respectively, and $X_{(11)}, X_{(21)}, \ldots, X_{(n 1)}$ are the order statistics of $X_{11}, X_{21}, \ldots, X_{n 1}$ in ascending order. Therefore, previous generalized point and confidence interval estimates could be obtained as well.

\section{Testing Problem}

In previous two sections, reliability analysis is discussed for MSR parameter $R_{s, k}$ under common and unequal location parameter cases. One may be interested in testing whether the strength and stress parameters $\alpha_{1}$ and $\alpha_{2}$ from Rayleigh distributions are equal. Here we present the likelihood ratio test to compare the equivalence between parameters $\alpha_{1}$ and $\alpha_{2}$.

The hypothesis test problem is stated as:

$$
H_{0}: \alpha_{1}=\alpha_{2}=\alpha \quad \text { vs } H_{1}: \alpha_{1} \neq \alpha_{2} .
$$

For large $n$, the likelihood ratio statistic has the following property:

$$
-2\left\{\ell_{2}\left(\hat{\lambda}_{1}, \hat{\lambda}_{2}, \hat{\alpha}\right)-\ell_{2}\left(\hat{\lambda}_{1}, \hat{\lambda}_{2}, \hat{\alpha}_{1}, \hat{\alpha}_{2}\right)\right\} \rightarrow \chi_{1}^{2} .
$$

Hence, the likelihood ratio test can be constructed by using the asymptotic distribution of $-2\left\{\ell_{2}\left(\hat{\lambda}_{1}, \hat{\lambda}_{2}, \hat{\alpha}\right)-\ell_{2}\left(\hat{\lambda}_{1}, \hat{\lambda}_{2}, \hat{\alpha}_{1}, \hat{\alpha}_{2}\right)\right\}$ and $H_{0}$ is rejected when $-2\left\{\ell_{2}\left(\hat{\lambda}_{1}, \hat{\lambda}_{2}, \hat{\alpha}\right)-\right.$ $\left.\ell_{2}\left(\hat{\lambda}_{1}, \hat{\lambda}_{2}, \hat{\alpha}_{1}, \hat{\alpha}_{2}\right)\right\}>c^{*}$, where $c^{*}$ satisfies $P\left(\chi_{1}^{2}>c^{*}\right)$ size of the test.

\section{Numerical Illustration}

\subsection{Simulation Studies}

In this section, simulation experiments are presented to evaluate the proposed methods. The performance of different estimates for MSR parameter $R_{s, k}$ is investigated by the following four criteria quantities:

- Mean square error (MSE) of different point estimates represented by $\bar{R}_{s, k}$, of $R_{s, k}$, which is computed by $\frac{1}{N} \sum\left(\bar{R}_{s, k}-R_{s, k}\right)^{2}$;

- Average absolute bias (AB) of different point estimates $\bar{R}_{s, k}$ of $R_{s, k}$, which is calculated as $\frac{1}{N} \sum\left|\bar{R}_{s, k}-R_{s, k}\right|$;

- Coverage probability (CP) of different $(1-\gamma) \%$ confidence intervals of $R_{s, k}$, which is defined as the probability that the estimated confidence interval contains the true value of the parameters;

- $\quad$ Average width (AW) of different $(1-\gamma) \%$ confidence intervals of the parameter $R_{s, k}$.

For different choices of Rayleigh parameters values $\lambda_{1}, \alpha_{1}, \lambda_{2}, \alpha_{2}$ and sample sizes $n, s, k$, the simulation was conducted for 10,000 runs. All aforementioned different estimates for MSR parameter $R_{s, k}$ were calculated, and the associated criteria quantities were obtained based on 10,000 simulation runs. The simulation results are reported in Table 1-4, where the significance level for interval estimates is given as $\gamma=0.95$. 
Table 1. ABs and MSEs (within bracket) for $R_{s, k}$ with common parameters.

\begin{tabular}{|c|c|c|c|c|c|}
\hline$\left(\lambda_{1}, \lambda_{2}, \alpha\right)$ & $(s, k)$ & $n$ & $\hat{R}_{s, k}$ & $\dot{R}_{s, k}$ & $\dot{R}_{s, k}^{F}$ \\
\hline \multirow[t]{8}{*}{$(1.5,0.8,2)$} & $(3,7)$ & 5 & 0.1142 [0.0296] & $0.1121[0.0271]$ & $0.1078[0.0263]$ \\
\hline & & 10 & 0.0817 [0.0161] & $0.0712[0.0152]$ & $0.0705[0.0143]$ \\
\hline & & 30 & $0.0785[0.0086]$ & $0.0602[0.0078]$ & $0.0582[0.0070]$ \\
\hline & & 50 & 0.0530 [0.0077] & 0.0483 [0.0036] & 0.0477 [0.0035] \\
\hline & $(5,10)$ & 5 & 0.1125 [0.0200] & 0.1059 [0.0172] & 0.1050 [0.0189] \\
\hline & & 10 & 0.0768 [0.0093] & $0.0701[0.0090]$ & $0.0698[0.0088]$ \\
\hline & & 30 & $0.0638[0.0072]$ & $0.0633[0.0065]$ & $0.0631[0.0062]$ \\
\hline & & 50 & 0.0473 [0.0054] & $0.0456[0.0033]$ & $0.0445[0.0030]$ \\
\hline \multirow[t]{8}{*}{$(1.8,1,1.5)$} & $(3,7)$ & 5 & $0.1260[0.0214]$ & 0.1117 [0.0198] & $0.1106[0.0186]$ \\
\hline & & 10 & $0.0897[0.0125]$ & 0.0809 [0.0103] & $0.0800[0.0100]$ \\
\hline & & 30 & $0.0674[0.0076]$ & 0.0579 [0.0057] & $0.0568[0.0053]$ \\
\hline & & 50 & $0.0483[0.0051]$ & $0.0477[0.0048]$ & $0.0473[0.0039]$ \\
\hline & $(5,10)$ & 5 & 0.1088 [0.0188] & 0.1048 [0.0169] & 0.1038 [0.0158] \\
\hline & & 10 & $0.0749[0.0112]$ & $0.0754[0.0088]$ & $0.0749[0.0086]$ \\
\hline & & 30 & $0.0642[0.0052]$ & $0.0537[0.0045]$ & $0.0535[0.0043]$ \\
\hline & & 50 & $0.0470[0.0046]$ & $0.0433[0.0036]$ & $0.0431[0.0030]$ \\
\hline
\end{tabular}

Table 2. AWs and CPs (within bracket) for $R_{s, k}$ with common parameters.

\begin{tabular}{|c|c|c|c|c|c|}
\hline \multirow{2}{*}{$\left(\lambda_{1}, \lambda_{2}, \alpha\right)$} & \multirow{2}{*}{$n$} & \multicolumn{2}{|l|}{$(s, k)=(3,7)$} & \multicolumn{2}{|l|}{$(s, k)=(5,10)$} \\
\hline & & ACI & GCI & ACI & GCI \\
\hline \multirow[t]{4}{*}{$(1.5,0.8,2)$} & 5 & $0.6243[0.6258]$ & 0.6168 [0.6633] & 0.4440 [0.7195] & $0.4347[0.7626]$ \\
\hline & 10 & $0.3421[0.7133]$ & $0.2554[0.7632]$ & $0.3241[0.8051]$ & $0.3176[0.8124]$ \\
\hline & 30 & 0.2074 [0.8127] & 0.1550 [0.8458] & $0.1398[0.8221]$ & $0.1106[0.8598]$ \\
\hline & 50 & $0.1583[0.8781]$ & $0.1236[0.8971]$ & $0.1168[0.9014]$ & $0.1034[0.9237]$ \\
\hline \multirow[t]{4}{*}{$(1.8,1,1.5)$} & 5 & $0.4633[0.7122]$ & 0.4624 [0.7857] & $0.3954[0.7763]$ & $0.3825[0.8766]$ \\
\hline & 10 & $0.4148[0.8272]$ & $0.6307[0.8531]$ & $0.3570[0.8844]$ & $0.3371[0.9074]$ \\
\hline & 30 & $0.2171[0.8788]$ & $0.2009[0.8874]$ & 0.1886 [0.8957] & $0.1648[0.9253]$ \\
\hline & 50 & $0.1546[0.9252]$ & 0.1354 [0.9229] & 0.1446 [0.9410] & $0.1265[0.9421]$ \\
\hline
\end{tabular}

Table 3. ABs and MSEs (within bracket) for $R_{s, k}$ with unequal parameters.

\begin{tabular}{|c|c|c|c|c|c|}
\hline$\left(\lambda_{1}, \alpha_{1}, \lambda_{2}, \alpha_{2}\right)$ & $(s, k)$ & $n$ & $\check{R}_{s, k}$ & $\grave{R}_{s, k}$ & $\grave{R}_{s, k}^{F}$ \\
\hline \multirow[t]{8}{*}{$(1.2,1.5,1.4,2)$} & $(3,7)$ & 5 & 0.1728 [0.0496] & 0.1086 [0.0413] & 0.0984 [0.0317] \\
\hline & & 10 & $0.1331[0.0333]$ & 0.0867 [0.0171] & $0.0849[0.0143]$ \\
\hline & & 30 & $0.0649[0.0094]$ & $0.0506[0.0063]$ & $0.0460[0.0054]$ \\
\hline & & 50 & $0.0445[0.0036]$ & $0.0309[0.0021]$ & $0.0306[0.0018]$ \\
\hline & $(5,10)$ & 5 & 0.1668 [0.0475] & 0.0863 [0.0188] & $0.0816[0.0100]$ \\
\hline & & 10 & $0.1557[0.0271]$ & $0.0663[0.0088]$ & $0.0610[0.0082]$ \\
\hline & & 30 & $0.0553[0.0076]$ & 0.0409 [0.0034] & $0.0394[0.0031]$ \\
\hline & & 50 & $0.0362[0.0025]$ & $0.0347[0.0022]$ & $0.0339[0.0020]$ \\
\hline \multirow[t]{8}{*}{$(1.6,1,1.5,1.2)$} & $(3,7)$ & 5 & 0.1618 [0.0477] & $0.1058[0.0245]$ & $0.0955[0.0210]$ \\
\hline & & 10 & $0.1226[0.0323]$ & $0.0728[0.0113]$ & $0.0681[0.0101]$ \\
\hline & & 30 & $0.0580[0.0089]$ & $0.0396[0.0028]$ & 0.0387 [0.0027] \\
\hline & & 50 & $0.0374[0.0028]$ & $0.0300[0.0015]$ & $0.0295[0.0015]$ \\
\hline & $(5,10)$ & 5 & 0.1557 [0.0456] & 0.0904 [0.0200] & $0.0786[0.0169]$ \\
\hline & & 10 & $0.1025[0.0251]$ & $0.0604[0.0084]$ & $0.0548[0.0074]$ \\
\hline & & 30 & $0.0450[0.0060]$ & $0.0315[0.0019]$ & $0.0303[0.0018]$ \\
\hline & & 50 & 0.0299 [0.0019] & $0.0231[0.0010]$ & $0.0225[0.0009]$ \\
\hline
\end{tabular}


Table 4. AWs and CPs (within bracket) for $R_{s, k}$ with unequal parameters.

\begin{tabular}{|c|c|c|c|c|c|}
\hline \multirow{2}{*}{$\left(\lambda_{1}, \lambda_{2}, \alpha_{1}, \alpha_{2}\right)$} & \multirow{2}{*}{$n$} & \multicolumn{2}{|l|}{$(s, k)=(3,7)$} & \multicolumn{2}{|l|}{$(s, k)=(5,10)$} \\
\hline & & ACI & GCI & ACI & GCI \\
\hline \multirow[t]{4}{*}{$(1.2,1.5,1.4,2)$} & 5 & $0.6625[0.7056]$ & $0.4336[0.7756]$ & $0.4728[0.7856]$ & $0.3799[0.8015]$ \\
\hline & 10 & $0.3743[0.8043]$ & $0.3527[0.8262]$ & $0.3677[0.8322]$ & $0.2581[0.8424]$ \\
\hline & 30 & $0.2662[0.8445]$ & $0.2520[0.8668]$ & $0.1425[0.8710]$ & $0.1150[0.8872]$ \\
\hline & 50 & $0.1415[0.8620]$ & $0.1382[0.8813]$ & $0.1355[0.8896]$ & $0.1042[0.9105]$ \\
\hline \multirow[t]{4}{*}{$(1.6,1,1.5,1.2)$} & 5 & 0.5477 [0.8419] & $0.3100[0.8552]$ & $0.3260[0.8473]$ & $0.2479[0.8574]$ \\
\hline & 10 & $0.2530[0.8623]$ & $0.2514[0.8686]$ & $0.1110[0.8662]$ & $0.1014[0.8893]$ \\
\hline & 30 & 0.1127 [0.8803] & $0.1110[0.8878]$ & $0.0984[0.9121]$ & 0.0910 [0.9269] \\
\hline & 50 & 0.1100 [0.8972] & 0.0814 [0.9010] & $0.0656[0.9271]$ & $0.0518[0.9355]$ \\
\hline
\end{tabular}

Based on the simulation results displayed in Table 1 and 3, it is observed that as the effective sample sizes $n$ or $(s, k)$ or their combination increase, ABs and MSEs of point estimates of MSR parameter $R_{s, k}$ decrease under both common and unequal location parameter cases, respectively. It verifies the consistency properties of all the proposed methods. One could also observe that both likelihood and pivotal estimates have a satisfactory performance in terms of ABs and MSEs. Under a fixed effective sample size, the pivotal quantities-based generalized point estimates $K^{R}$ and $\tilde{R}^{F}$ have smaller ABs and MSEs than those of MLEs $\hat{R}$ under a common location parameter case where $\grave{R}^{F}$ is superior to $\hat{R}$. Similar phenomenon also appears among estimates $\grave{R} \grave{R}^{F}$, and $\breve{R}$ under an unequal parameter case in term of ABs and MSEs. Taking Table 2 and 4 into consideration, one also observed that the AWs of ACIs and GCIs are getting smaller as the effective sample size increases, and correspondingly the associated CPs increase when the sample size increases under both common and unequal location parameter cases. In general, the CPs of the proposed GCIs are more close to the nominal level than ACIs. Under a fixed sample size, the GCIs have smaller AWs and larger CPs than those of ACIs in most situations. Therefore, one can draw conclusions from this simulation results that the pivotal estimates perform better than conventional likelihood-based point and interval results in terms of criteria quantities.

\subsection{Real Data Illustration}

In this section, the monthly water capacity of the Shasta reservoir in California, USA during the months of August, September, and December from 1980 to 2015 (see http:/ / cdec.water.ca.gov/ cgi-progs/queryMonthly?SHA, accessed on 19 September 2021) is discussed to illustrate the utilization of the proposed procedures. Suppose that $k=5$, $n=6, Y_{1}$ is the capacities of December 1980, and $X_{11}, \ldots, X_{15}$ are the capacities of August from 1981 to 1985; $Y_{2}$ is the capacities of December 1986, $X_{21}, \ldots, X_{25}$ are the capacities of August from 1987 to 1991 and so on. For illustration, all of the values are divided by $3,014,878$ (the mean of water capacity) and the transformed data are obtained as:

Observed complete strength data

$\left(\begin{array}{lllll}0.4238 & 0.5579 & 0.7262 & 0.8112 & 0.8296 \\ 0.2912 & 0.3634 & 0.3719 & 0.4637 & 0.4785 \\ 0.5381 & 0.5612 & 0.7226 & 0.7449 & 0.7540 \\ 0.5249 & 0.6060 & 0.6686 & 0.7159 & 0.7552 \\ 0.3451 & 0.4253 & 0.4688 & 0.7188 & 0.7420 \\ 0.2948 & 0.3929 & 0.4616 & 0.6139 & 0.7951\end{array}\right)$

Observed complete stress data

and $\quad\left(\begin{array}{c}0.7009 \\ 0.6532 \\ 0.4589 \\ 0.7183 \\ 0.5310 \\ 0.7665\end{array}\right)$

For the above-transformed data, one can refer to Kizilaslan and Nadar [11] for more details, whereas the complete monthly water capacity of the Shasta reservoir in California, USA between 1981 to 1985 is provided in Appendix G.

Before progressing further, we check whether the Rayleigh distribution could be used to fit these real life data. The Kolmogorov-Smirnov (K-S) distances and the corresponding $p$-values (within brackets) are $0.1754(0.2802)$ and $0.2703(0.6841)$, respectively. Moreover, 
the empirical cumulative distributions plot and overlay the theoretical Rayleigh distribution, the probability-probability (P-P) and the Quantile-Quantile (Q-Q) plots are shown in Figure 1, which also imply that the Rayleigh distribution can be used as a proper model to fit this dataset. Therefore, it is noted that the Rayleigh distribution fits the transformed dataset well and can be used as a proper model.
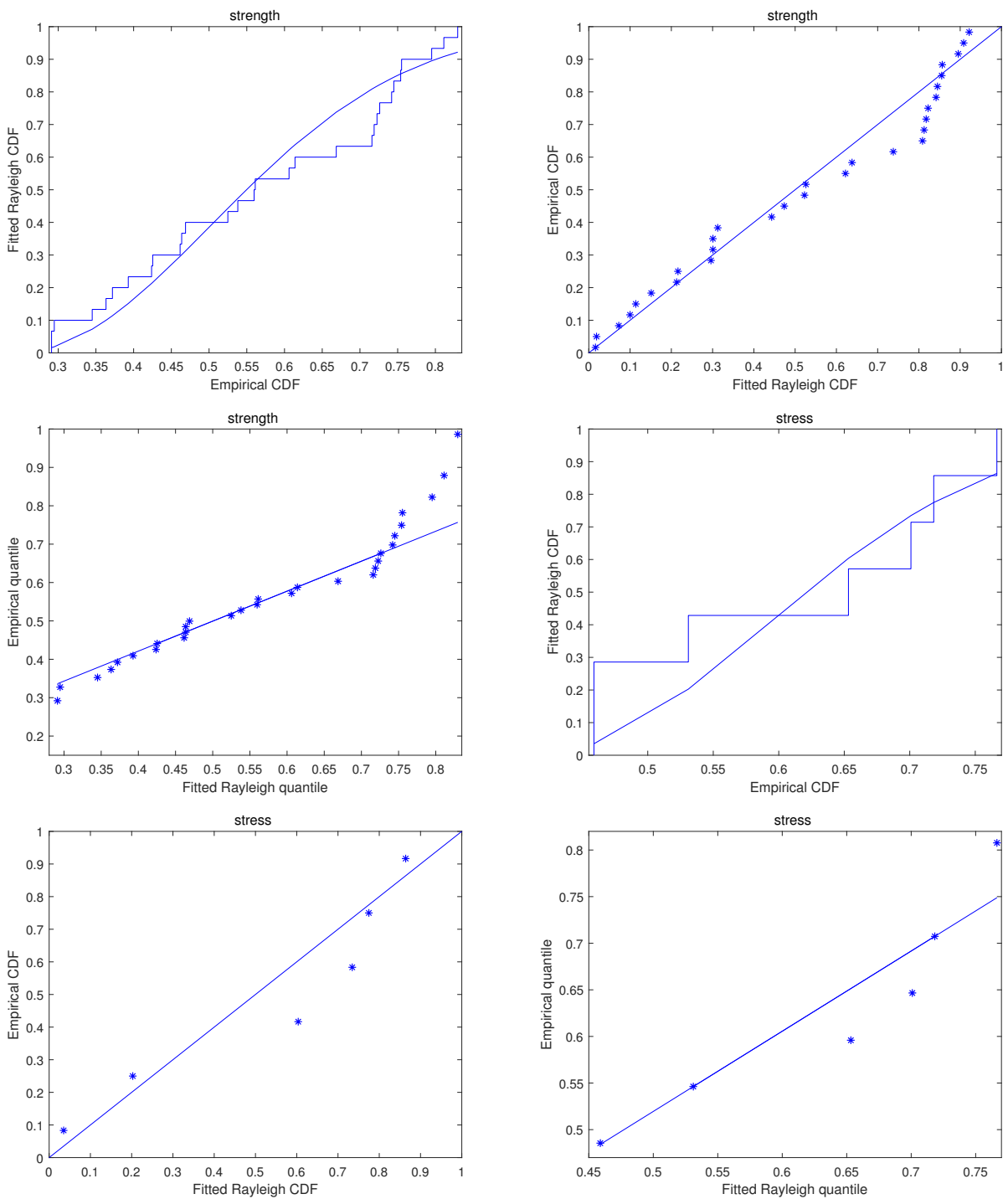

Figure 1. Empirical distribution and fitted Rayleigh distribution, P-P, and Q-Q plots based on real data.

Based on previous complete strength and stress data, a MSR censored observation of the 3-out-of-5 G system can be constructed as:

\begin{tabular}{|c|c|c|c|c|}
\hline \multicolumn{3}{|c|}{ Strength data of $X$} & \multirow{2}{*}{\multicolumn{2}{|c|}{ Stress data of 1}} \\
\hline 0.4238 & 0.5579 & 0.7262 & & \\
\hline 0.2912 & 0.3634 & 0.3719 & & 0.6532 \\
\hline 0.5381 & 0.5612 & 0.7226 & & 0.4589 \\
\hline 0.5249 & 0.6060 & 0.6686 & and & 0.7183 \\
\hline 0.3451 & 0.4253 & 0.4688 & & 0.5310 \\
\hline 0.2948 & 0.3929 & 0.4616 & & $0.7665)$ \\
\hline
\end{tabular}

The associated point and interval estimates for MSR parameter $R_{s, k}$ are presented in Table 5 under both common and unequal location parameter cases, where the significance 
level $\gamma$ is taken to be 0.05 and the associated interval lengths are obtained in squared brackets. It is observed that the point estimates are close to each other and the GCIs of $R_{s, k}$ perform better than associated ACIs in terms of interval length.

Table 5. Estimates of $R_{s, k}$ based on the real data set.

\begin{tabular}{lllll}
\hline \multicolumn{5}{c}{ Common Parameter Case } \\
\hline$\hat{R}_{s, k}$ & $\dot{R}_{s, k}$ & $\dot{R}_{s, k}^{F}$ & ACI & GCI \\
\hline 0.4766 & 0.5208 & 0.4971 & $(0.4551,0.5231)[0.0680]$ & $(0.4687,0.4995)[0.0308]$ \\
\hline \multicolumn{5}{c}{ Unequal parameter case } \\
\hline$\check{R}_{s, k}$ & $\grave{R}_{s, k}$ & $\grave{R}_{s, k}^{F}$ & ACI & GCI \\
\hline 0.5525 & 0.5662 & 0.5776 & $(0.4439,0.6532)[0.2093]$ & $(0.4851,0.6530)[0.1579]$ \\
\hline
\end{tabular}

Moreover, for comparing the equivalence between strength and stress location parameters, $\alpha_{1}$ and $\alpha_{2}$, the likelihood ratio statistic and $p$-value are 7.4508 and 0.0063 , respectively. There is sufficient statistical evidence to reject the null hypothesis $\alpha_{1}=\alpha_{2}$ at a 0.05 significance level. Therefore, it is recommended that the strength and stress are from Rayleigh distributions with unequal parameters $\alpha_{1}$ and $\alpha_{2}$ under this monthly capacity data.

\section{Concluding Remarks}

Inference for a multicomponent stress-strength model is studied when the latent strength and stress random variables follow Rayleigh distributions in this paper. The existence and uniqueness of the MLE for MSR parameter $R_{s, k}$ have been established under common and unequal location parameter situations. ACIs are also provided by using a large sample theory and delta technique. Alternatively, a series of pivotal quantities are constructed and correspondingly the generalized pivotal-based estimators for the Rayleigh MSR parameter $R_{s, k}$ are proposed as well. From simulation results and the real life example, it is observed that the proposed procedures work quite well under the given sampling scheme, and that the proposed pivotal quantities-based generalized estimates perform better than conventional likelihood-based point and confidence interval estimates under both common and unequal location parameter cases, respectively. Although the inference is discussed under Type-II censored data, the methodologies proposed in this work can be extended to other data types, such as complete data, progressively Type-II censored data, and progressively first-failure Type-II censored data with proper modification.

Author Contributions: All authors equally contributed in writing this manuscript. All authors have read and agreed to the published version of the manuscript.

Funding: This research was funded by the National Natural Science Foundation of China (no. 12061091), the Yunnan Fundamental Research Projects (no. 202101AT070103), and the Doctoral Research Foundation of Yunnan Normal University (no. 00800205020503129).

Institutional Review Board Statement: Not applicable.

Informed Consent Statement: Not applicable.

Data Availability Statement: Complete monthly water capacity data of the Shasta Reservoir from 1981 to 1985 is given in Appendix G. The observed complete strength data and observed complete stress data were given in the first paragraph in Section 6.2.

Acknowledgments: Authors would like to thank the editor and reviewers for their helpful comments and suggestions, which improved this paper significantly.

Conflicts of Interest: The authors declare no conflict of interest. 


\section{Appendix A. The Proof of Theorem 1}

For fixed $n, s, k$ and given $\alpha$, taking derivatives of $\ell_{1}\left(\lambda_{1}, \lambda_{2}, \alpha\right)$ with respect to parameters $\lambda_{1}, \lambda_{2}$ and setting them to zero, one can obtain the conditional MLEs $\dot{\lambda}_{1}, \dot{\lambda}_{2}$ of $\lambda_{1}, \lambda_{2}$ directly. Moreover, using inequality $\ln t \leq t-1$ for $t=\frac{\lambda_{i}}{\lambda_{i}}(>0), i=1,2$, one has:

$$
\ln \lambda_{1} \leq \frac{\lambda_{1}}{n s}\left[\sum_{i=1}^{n} \sum_{j=1}^{s}\left(x_{i j}-\alpha\right)^{2}+(k-s) \sum_{i=1}^{n}\left(x_{i s}-\alpha\right)^{2}\right]-1+\ln \dot{\lambda}_{1}
$$

and

$$
\ln \lambda_{2} \leq \frac{\lambda_{2}}{n}\left[\sum_{i=1}^{n}\left(y_{i}-\alpha\right)^{2}\right]-1+\ln \dot{\lambda}_{2}
$$

Ignoring the constant terms and substituting inequalities (A1) and (A2) into the loglikelihood function (7), one further has:

$$
\begin{aligned}
\ell_{1}\left(\lambda_{1}, \lambda_{2}, \alpha\right) \leq & \lambda_{1}\left\{\sum_{i=1}^{n} \sum_{j=1}^{s}\left(x_{i j}-\alpha\right)^{2}+(k-s) \sum_{i=1}^{n}\left(x_{i s}-\alpha\right)^{2}\right\}-n s+n s \ln \dot{\lambda}_{1} \\
& +\lambda_{2} \sum_{i=1}^{n} \ln \left(y_{i}-\alpha\right)^{2}-n+n \ln \dot{\lambda}_{2}+\sum_{i=1}^{n} \sum_{j=1}^{s} \ln \left(x_{i j}-\alpha\right)+\sum_{i=1}^{n} \ln \left(y_{i}-\alpha\right) \\
& -\lambda_{1}\left(\sum_{i=1}^{n} \sum_{j=1}^{s}\left(x_{i j}-\alpha\right)^{2}+(k-s) \sum_{i=1}^{n}\left(x_{i s}-\alpha\right)^{2}\right)-\lambda_{2} \sum_{i=1}^{n}\left(y_{i}-\alpha\right)^{2} .
\end{aligned}
$$

Since,

$$
n s=\dot{\lambda}_{1}\left\{\sum_{i=1}^{n} \sum_{j=1}^{s}\left(x_{i j}-\alpha\right)^{2}+(k-s) \sum_{i=1}^{n}\left(x_{i s}-\alpha\right)^{2}\right\} \quad \text { and } n=\dot{\lambda}_{2} \sum_{i=1}^{n}\left(y_{i}-\alpha\right)^{2},
$$

following inequality can be obtained as:

$$
\begin{aligned}
& \ell_{1}\left(\lambda_{1}, \lambda_{2}, \alpha\right) \leq n s \ln \dot{\lambda}_{1}+n \ln \dot{\lambda}_{2}+\sum_{i=1}^{n} \sum_{j=1}^{s} \ln \left(x_{i j}-\alpha\right)+\sum_{i=1}^{n} \ln \left(y_{i}-\alpha\right) \\
& -\dot{\lambda}_{1}\left(\sum_{i=1}^{n} \sum_{j=1}^{s}\left(x_{i j}-\alpha\right)^{2}+(k-s) \sum_{i=1}^{n}\left(x_{i s}-\alpha\right)^{2}\right)-\dot{\lambda}_{2} \sum_{i=1}^{n}\left(y_{i}-\alpha\right)^{2} \\
& =\ell_{1}\left(\dot{\lambda}_{1}, \dot{\lambda}_{2}, \alpha\right) .
\end{aligned}
$$

Equality holds iff $\lambda_{1}=\dot{\lambda}_{1}$ and $\lambda_{2}=\dot{\lambda}_{2}$. This completes the proof.

\section{Appendix B. The Proof of Theorem 2}

Based on (9), taking derivatives of $\ell_{11}(\alpha)$ and setting it to zero, one has:

$$
\begin{aligned}
\frac{\partial \ell_{11}(\alpha)}{\partial \alpha} & =-\sum_{i=1}^{n} \sum_{j=1}^{s} \frac{1}{x_{i j}-\alpha}-\sum_{i=1}^{n} \frac{1}{y_{i}-\alpha}+\frac{2 n \sum_{i=1}^{n}\left(y_{i}-\alpha\right)}{\sum_{i=1}^{n}\left(y_{i}-\alpha\right)^{2}} \\
& +\frac{2 n s\left[\sum_{i=1}^{n} \sum_{j=1}^{s}\left(x_{i j}-\alpha\right)+(k-s) \sum_{i=1}^{n}\left(x_{i s}-\alpha\right)\right]}{\sum_{i=1}^{n} \sum_{j=1}^{s}\left(x_{i j}-\alpha\right)^{2}+(k-s) \sum_{i=1}^{n}\left(x_{i s}-\alpha\right)^{2}}=0,
\end{aligned}
$$

then the result is obtained. 
Ignoring the constant terms, we write (7) as:

$$
\begin{aligned}
\ell_{1}\left(\lambda_{1}, \lambda_{2}, \alpha\right) & =n s \ln \lambda_{1}+n \ln \lambda_{2}+\sum_{i=1}^{n} \sum_{j=1}^{s} \ln \left(x_{i j}-\alpha\right)+\sum_{i=1}^{n} \ln \left(y_{i}-\alpha\right) \\
& -\lambda_{1}\left(\sum_{i=1}^{n} \sum_{j=1}^{s}\left(x_{i j}-\alpha\right)^{2}+(k-s) \sum_{i=1}^{n}\left(x_{i s}-\alpha\right)^{2}\right)-\lambda_{2} \sum_{i=1}^{n}\left(y_{i}-\alpha\right)^{2} .
\end{aligned}
$$

From the above expression, it is observed that the domain of $\lambda_{1}, \lambda_{2}, \alpha$ is defined as $(0,+\infty) \times(0, \infty) \times\left(0, t_{0}\right)$ with $t_{0}=\min \left\{\min \left\{x_{i 1}\right\}_{i=1}^{n}, \min \left\{y_{i}\right\}_{i=1}^{n}\right\}$. Next, we will show the maximum of $\ell_{1}\left(\lambda_{1}, \lambda_{2}, \alpha\right)$ exists and is unique in $(0,+\infty) \times(0, \infty) \times\left(-\infty, t_{0}\right)$. Regarding this, it is observed that:

$$
\begin{aligned}
\frac{\partial^{2} \ell_{1}\left(\lambda_{1}, \lambda_{2}, \alpha\right)}{\partial \lambda_{1}^{2}} & =-\frac{n s}{\lambda_{1}^{2}}<0, \frac{\partial^{2} \ell_{1}\left(\lambda_{1}, \lambda_{2}, \alpha\right)}{\partial \lambda_{2}^{2}}=-\frac{n}{\lambda_{1}^{2}}<0, \\
\frac{\partial^{2} \ell_{1}\left(\lambda_{1}, \lambda_{2}, \alpha\right)}{\partial \alpha^{2}} & =-\sum_{i=1}^{n} \sum_{j=1}^{s} \frac{1}{\left(x_{i j}-\alpha\right)^{2}}-\sum_{i=1}^{n} \frac{1}{\left(y_{i}-\alpha\right)^{2}} \\
& -2 \lambda_{1}[(n-1) s+k]-2 \lambda_{2} n<0 .
\end{aligned}
$$

Therefore, for fixed $\lambda_{1}(\alpha)$ and $\lambda_{2}(\alpha), \ell_{1}\left(\lambda_{1}, \lambda_{2}, \alpha\right)$ is a strictly concave function of $\alpha\left(\lambda_{1}, \lambda_{2}\right)$.

Furthermore, for fixed $\lambda_{1}(\alpha)$ and $\lambda_{2}(\alpha)$, it is noted that:

$$
\lim _{\alpha \rightarrow-\infty} \ell_{1}\left(\lambda_{1}, \lambda_{2}, \alpha\right)=-\infty \text { and } \lim _{\alpha \rightarrow t_{0}} \ell_{1}\left(\lambda_{1}, \lambda_{2}, \alpha\right)=-\infty
$$

and for fixed $\alpha$ one also has:

$$
\begin{aligned}
& \lim _{\lambda_{1} \rightarrow 0} \ell_{1}\left(\lambda_{1}, \lambda_{2}, \alpha\right)=-\infty, \quad \lim _{\lambda_{1} \rightarrow+\infty} \ell_{1}\left(\lambda_{1}, \lambda_{2}, \alpha\right)=-\infty, \\
& \lim _{\lambda_{2} \rightarrow 0} \ell_{1}\left(\lambda_{1}, \lambda_{2}, \alpha\right)=-\infty, \quad \lim _{\lambda_{2} \rightarrow+\infty} \ell_{1}\left(\lambda_{1}, \lambda_{2}, \alpha\right)=-\infty .
\end{aligned}
$$

Therefore, $\ell_{1}\left(\lambda_{1}, \lambda_{2}, \alpha\right)$ is an unimodal function with respect to $\alpha$ for given $\lambda_{1}(\alpha)$ and $\lambda_{2}(\alpha)$. Furthermore, it is seen that:

$$
\begin{aligned}
& \lim _{\substack{\lambda_{1} \rightarrow 0, \lambda_{2} \rightarrow 0, \alpha \rightarrow-\infty}} \ell_{1}\left(\lambda_{1}, \lambda_{2}, \alpha\right)=-\infty, \lim _{\substack{\lambda_{1} \rightarrow 0, \lambda_{1} \rightarrow 0, \alpha \rightarrow t_{0}}} \ell_{1}\left(\lambda_{1}, \lambda_{2}, \alpha\right)=-\infty, \\
& \lim _{\substack{\lambda_{1} \rightarrow+\infty, \lambda_{2} \rightarrow+\infty \\
\alpha \rightarrow-\infty}} \ell_{1}\left(\lambda_{1}, \lambda_{2}, \alpha\right)=-\infty, \lim _{\substack{\lambda_{1} \rightarrow+\infty, \lambda_{1} \rightarrow+\infty \\
\alpha \rightarrow t_{0}}} \ell_{1}\left(\lambda_{1}, \lambda_{2}, \alpha\right)=-\infty,
\end{aligned}
$$
the set:

Suppose $\left(\lambda_{1}^{0}, \lambda_{2}^{0}, \alpha^{0}\right) \in(0, \infty) \times(0, \infty) \times\left(-\infty, t_{0}\right)$ and $l\left(\lambda_{1}^{0}, \lambda_{2}^{0}, \alpha^{0}\right)=d$. Consider

$$
C=\left\{\left(\lambda_{1}, \lambda_{2}, \alpha\right):\left(\lambda_{1}, \lambda_{2}, \alpha\right) \in(0, \infty) \times(0, \infty) \times\left(-\infty, t_{0}\right), l\left(\lambda_{1}, \lambda_{2}, \alpha\right) \geq d\right\} .
$$

Obviously, $C$ is a closed and bounded set, then set $C$ is compact. Since $\ell_{1}\left(\lambda_{1}, \lambda_{2}, \alpha\right)$ is a continuous function of parameters $\lambda_{1}, \lambda_{2}$, and $\alpha$. Therefore, $\ell_{1}\left(\lambda_{1}, \lambda_{2}, \alpha\right)$ has a maximum in set $C$ denoted $\ell_{1}\left(\lambda_{1}^{m}, \lambda_{2}^{m}, \alpha^{m}\right)$ at parameters $\left(\lambda_{1}^{m}, \lambda_{2}^{m}, \alpha^{m}\right)$. Next, we will show $\left(\lambda_{1}^{m}, \lambda_{2}^{m}, \alpha^{m}\right)$ is unique. Note that for $\left(\lambda_{1}, \lambda_{2}, \alpha\right) \in(0, \infty) \times(0, \infty) \times\left(-\infty, t_{0}\right)$, one respectively has:

$$
\ell_{1}\left(\lambda_{1}^{m}, \lambda_{2}^{m}, \alpha^{m}\right)>\ell_{1}\left(\lambda_{1}^{m}, \lambda_{2}, \alpha^{m}\right)>\ell_{1}\left(\lambda_{1}, \lambda_{2}, \alpha^{m}\right)>\ell_{1}\left(\lambda_{1}, \lambda_{2}, \alpha\right) \text { for } \alpha^{m}>0 \text {, }
$$

and

$$
\ell_{1}\left(\lambda_{1}^{m}, \lambda_{2}^{m}, \alpha^{m}\right)>\ell_{1}\left(\lambda_{1}^{m}, \lambda_{2}^{m}, 0\right)>\ell_{1}\left(\lambda_{1}^{m}, \lambda_{2}^{m}, \mu\right)>\ell_{1}\left(\lambda_{1}, \lambda_{2}, \mu\right) \quad \text { for } \quad \alpha^{m} \leq 0,
$$


where the MLE of $\left(\lambda_{1}, \lambda_{2}, \alpha\right)$ reduces to $\left(\lambda_{1}, \lambda_{2}, 0\right)$ for the second case and is unique. Therefore, the assertion is completed.

Appendix C. The Proof of Theorem 4

Based on Theorem 1 and delta method [21], $R_{s, k}(\hat{\eta})$ can be written as:

$$
\begin{aligned}
R_{s, k}(\hat{\eta}) & =R_{s, k}(\eta)+\left(\frac{\partial R_{s, k}(\eta)}{\partial \eta}\right)^{T}(\hat{\eta}-\eta)+\frac{1}{2}(\hat{\eta}-\eta)^{T}\left(\frac{\partial^{2} R_{s, k}\left(\eta^{*}\right)}{\partial \eta}\right)(\hat{\eta}-\eta) \\
& \approx R_{s, k}(\eta)+\left(\frac{\partial R_{s, k}(\eta)}{\partial \eta}\right)^{T}(\hat{\eta}-\eta)
\end{aligned}
$$

using the Taylor series expansion and the differential mean value theorem, where $\frac{\partial R_{s, k}(\eta)}{\partial \eta}$ and $\frac{\partial^{2} R_{s, k}(\eta)}{\partial \eta}$ denotes the matrices of the first and second derivatives for $R_{s, k}$ with respect to $\eta$, and $\eta^{*}$ is some proper value between $\eta$ and $\hat{\eta}$. Then expression (A3) could be rewritten as:

$$
R_{s, k}(\hat{\eta})-R_{s, k}(\eta) \approx\left(\frac{\partial R_{s, k}(\eta)}{\partial \eta}\right)^{T}(\hat{\eta}-\eta),
$$

which implies that $R_{s, k}(\hat{\eta}) \rightarrow R_{s, k}(\eta)$ when $n \rightarrow \infty$ by using $\hat{\eta} \rightarrow \eta$ from Theorem 1 . Moreover, from (A3), since the variance of $R_{s, k}(\hat{\eta})$ can be written as:

$$
\begin{aligned}
\operatorname{Var}\left[R_{s, k}(\hat{\eta})\right] & \approx \operatorname{Var}\left[R_{s, k}(\eta)+\left(\frac{\partial R_{s, k}(\eta)}{\partial \eta}\right)^{T} \hat{\eta}-\left(\frac{\partial R_{s, k}(\eta)}{\partial \eta}\right)^{T} \eta\right] \\
& =\operatorname{Var}\left[\left(\frac{\partial R_{s, k}(\eta)}{\partial \eta}\right)^{T} \hat{\eta}\right]=\left(\frac{\partial R_{s, k}(\eta)}{\partial \eta}\right)^{T} \operatorname{Var}[\hat{\eta}]\left(\frac{\partial R_{s, k}(\eta)}{\partial \eta}\right) .
\end{aligned}
$$

Therefore, using the central limit theory and Theorem 1, one has:

$$
R_{s, k}(\hat{\eta})-R_{s, k}(\eta) \stackrel{d}{\rightarrow} N\left(0,\left(\frac{\partial R_{s, k}(\eta)}{\partial \eta}\right)^{T} \operatorname{Var}[\hat{\eta}]\left(\frac{\partial R_{s, k}(\eta)}{\partial \eta}\right)\right),
$$

and the assertion is shown by normalization.

\section{Appendix D. The Proof of Theorem 5}

For $0<\alpha<\min \left\{\min \left\{x_{i 1}\right\}_{i=1}^{n}, y_{(1)}\right\}$, since $X_{i 1}, X_{i 2}, \ldots, X_{i s}$ are the first $s$ order statistics of size $k$ from Rayleigh distribution, then quantities $T_{i j}=\lambda_{1}\left(X_{i j}-\alpha\right)^{2}, j=1,2, \ldots, s$ can be viewed as the Type-II censored data from standard exponential distribution with mean one. Using the lack of memory property of the standard exponential distribution, it is seen that quantities $Z_{i 1}=k T_{i 1}, Z_{i 2}=(k-1)\left(T_{i 2}-T_{i 1}\right), \cdots, Z_{i s}=(k-s+1)\left(T_{i s}-T_{i(s-1)}\right)$ are i.i.d. samples from the standard exponential distribution with mean 1 (see Lawless [22]).

For $i=1,2, \ldots, n$, let $W_{i j}=\sum_{r=1}^{j} Z_{i r}=\lambda_{1}\left\{(k-j)\left(X_{i j}-\alpha\right)^{2}+\sum_{r=1}^{j}\left(X_{i r}-\alpha\right)^{2}\right\}$, $j=1,2, \ldots, s$, one could conduct from Stephens [23] and Viveros and Balakrishnan [24] that $U_{i 1}=\frac{W_{i 1}}{W_{i s}}, U_{i 2}=\frac{W_{i 2}}{W_{i s}}, \ldots, U_{i(s-1)}=\frac{W_{i(s-1)}}{W_{i s}}$ are order statistics from the uniform distribution between 0 to 1 with sample size $s-1$. Moreover, $U_{i 1}<U_{i 2}<\cdots<U_{i(s-1)}$ are also independent with $W_{i s}=\sum_{r=1}^{s} Z_{i r}=\lambda_{1}\left\{(k-s)\left(X_{i s}-\alpha\right)^{2}+\sum_{r=1}^{s}\left(X_{i r}-\alpha\right)^{2}\right\}$.

Using theory of sampling distribution, it is observed directly that quantity $P_{i 1}(\alpha)=-2 \sum_{j=1}^{s-1} \ln \left(U_{i j}\right)$ follows a chi-square distribution with $2(s-1)$ degrees of freedom, which is independent with $Q_{i 1}\left(\lambda_{1}, \alpha\right)=2 W_{i s}$ being chi-square distributed with $2 s$ degrees of freedom. Therefore, using the independent property of $P_{i 1}(\alpha), P_{i 2}(\alpha), \ldots, P_{i n}(\alpha)$, one has that: 


$$
P_{1}^{X}(\alpha)=2 \sum_{i=1}^{n} P_{i 1}(\alpha)=2 \sum_{i=1}^{n} \sum_{j=1}^{s-1} \ln \left[\frac{(k-s)\left(X_{i s}-\alpha\right)^{2}+\sum_{r=1}^{s}\left(X_{i r}-\alpha\right)^{2}}{(k-j)\left(X_{i s}-\alpha\right)^{2}+\sum_{r=1}^{j}\left(X_{i r}-\alpha\right)^{2}}\right]
$$

comes from a chi-square distribution with $2 n(s-1)$ degrees of freedom, and:

$$
Q_{1}^{X}\left(\lambda_{1}, \alpha\right)=2 \sum_{i=1}^{n} Q_{i 1}\left(\lambda_{1}, \alpha\right)=2 \lambda_{1} \sum_{i=1}^{n}\left\{(k-s)\left(X_{i s}-\alpha\right)^{2}+\sum_{r=1}^{s}\left(X_{i r}-\alpha\right)^{2}\right\} .
$$

follows chi-square distribution with $2 n s$ degrees of freedom. $P_{1}^{X}(\alpha)$ and $Q_{1}^{X}\left(\lambda_{1}, \alpha\right)$ are statistically independent. Therefore, the assertion is completed.

\section{Appendix E. The Proof of Theorem 6}

Let $Y_{(1)}, Y_{(2)}, \ldots, Y_{(n)}$ be the corresponding order statistics of stress random variables $Y_{1}, Y_{2}, \ldots, Y_{n}$. Then $\lambda_{2}\left(Y_{(1)}-\alpha\right)^{2}, \lambda_{2}\left(Y_{(2)}-\alpha\right)^{2}, \ldots, \lambda_{2}\left(Y_{(n)}-\alpha\right)^{2}$ are order statistics from standard exponential distribution with mean one. Following the similar line of Theorem 5 , The results can be shown similarly and details are omitted here for sake of concision.

\section{Appendix F. The Proof of Corollary 1}

Using the definitions of notations $P_{1}^{X}$ and $P_{1}^{Y}$, one has:

$$
\frac{(k-s)\left(X_{i s}-\alpha\right)^{2}+\sum_{r=1}^{s}\left(X_{i r}-\alpha\right)^{2}}{(k-j)\left(X_{i j}-\alpha\right)^{2}+\sum_{r=1}^{j}\left(X_{i r}-\alpha\right)^{2}}=1+\frac{(k-s)\left[\frac{X_{i s}-\alpha}{X_{i j}-\alpha}\right]^{2}+\sum_{r=j+1}^{s}\left[\frac{X_{i r}-\alpha}{X_{i j}-\alpha}\right]^{2}-(k-j)}{\sum_{r=1}^{j}\left[\frac{X_{i r}-\alpha}{X_{i j}-\alpha}\right]^{2}+(k-j)},
$$

and

$$
\frac{\sum_{r=1}^{n}\left[Y_{(r)}-\alpha\right]^{2}}{(n-j)\left[Y_{(j)}-\alpha\right]^{2}+\sum_{r=1}^{j}\left[Y_{(r)}-\alpha\right]^{2}}=1+\frac{\left[\frac{Y_{(n)}-\alpha}{Y_{(j)}-\alpha}\right]^{2}+\sum_{r=j+1}^{n}\left[\frac{Y_{(r)}-\alpha}{Y_{(j)}-\alpha}\right]^{2}-(n-j)}{\sum_{r=1}^{j}\left[\frac{Y_{(r)}-\alpha}{Y_{(j)}-\alpha}\right]^{2}+(n-j)} .
$$

From Lemma 1, it is seen that the numerators of above expressions increase in $\alpha$ and the associated denominators decrease in $\alpha$. Therefore, pivotal quantities $P_{1}^{X}(\alpha)$ and $P_{1}^{Y}(\alpha)$ are increasing functions on $\alpha$.

\section{Appendix G. Complete Monthly Water Capacity Data of the Shasta Reservoir}

Table A1. The capacity data of Shasta reservoir from the years 1981 to 1985.

\begin{tabular}{lc|cc|cc}
\hline DATE & STORAGE AF & DATE & STORAGE AF & DATE & STORAGE AF \\
\hline $01 / 1981$ & $3,453,500$ & $09 / 1982$ & $3,486,400$ & $05 / 1984$ & $4,294,400$ \\
$02 / 1981$ & $3,865,200$ & $10 / 1982$ & $3,433,400$ & $06 / 1984$ & $4,070,000$ \\
$03 / 1981$ & $4,320,700$ & $11 / 1982$ & $3,297,100$ & $07 / 1984$ & $3,587,400$ \\
$04 / 1981$ & $4,295,900$ & $12 / 1982$ & $3,255,000$ & $08 / 1984$ & $3,305,500$ \\
$05 / 1981$ & $3,994,300$ & $01 / 1983$ & $3,740,300$ & $09 / 1984$ & $3,240,100$ \\
$06 / 1981$ & $3,608,600$ & $02 / 1983$ & $3,579,400$ & $10 / 1984$ & $3,155,400$ \\
$07 / 1981$ & $3,033,000$ & $03 / 1983$ & $3,725,100$ & $11 / 1984$ & $3,252,300$ \\
$08 / 1981$ & $2,547,600$ & $04 / 1983$ & $4,286,100$ & $12 / 1984$ & $3,105,500$ \\
$09 / 1981$ & $2,480,200$ & $05 / 1983$ & $4,526,800$ & $01 / 1985$ & $3,118,200$ \\
$10 / 1981$ & $2,560,200$ & $06 / 1983$ & $4,471,200$ & $02 / 1985$ & $3,240,400$ \\
$11 / 1981$ & $3,336,700$ & $07 / 1983$ & $4,169,900$ & $03 / 1985$ & $3,445,500$ \\
$12 / 1981$ & $3,492,000$ & $08 / 1983$ & $3,776,200$ & $04 / 1985$ & $3,546,900$ \\
$01 / 1982$ & $3,556,300$ & $09 / 1983$ & $3,616,800$ & $05 / 1985$ & $3,225,400$ \\
\hline
\end{tabular}


Table A1. Cont.

\begin{tabular}{lc|lc|lc}
\hline DATE & STORAGE AF & DATE & STORAGE AF & DATE & STORAGE AF \\
\hline $02 / 1982$ & $3,633,500$ & $10 / 1983$ & $3,458,000$ & $06 / 1985$ & $2,856,300$ \\
$03 / 1982$ & $4,062,000$ & $11 / 1983$ & $3,395,400$ & $07 / 1985$ & $2,292,100$ \\
$04 / 1982$ & $4,472,700$ & $12 / 1983$ & $3,457,500$ & $08 / 1985$ & $1,929,200$ \\
$05 / 1982$ & $4,507,500$ & $01 / 1984$ & $3,405,200$ & $09 / 1985$ & $1,977,800$ \\
$06 / 1982$ & $4,375,400$ & $02 / 1984$ & $3,789,900$ & $10 / 1985$ & $2,083,100$ \\
$07 / 1982$ & $4,071,200$ & $03 / 1984$ & $4,133,600$ & $11 / 1985$ & $2,173,900$ \\
$08 / 1982$ & $3,692,400$ & $04 / 1984$ & $4,342,700$ & $12 / 1985$ & $2,422,100$
\end{tabular}

The website: https://cdec.water.ca.gov/dynamicapp/QueryMonthly?s=SHA, (accessed on 19 September 2021)

\section{References}

1. Eryilmaz, S. Phase type stress-strength models with reliability applications. Commun.-Stat.-Simul. Comput. 2018, 47, 954-963. [CrossRef]

2. Kundu, D.; Kundu, D. Estimation of $\mathrm{R}=\mathrm{P}(\mathrm{Y}<\mathrm{X})$ for three-parameter Weibull distribution. Stat. Prob. Lett. 2009, 79, $1839-1846$.

3. Krishnamoorthy, K.; Lin, Y. Confdence limits for stress-strength reliability involving Weibull models. J. Stat. Plan. Infer. 2010, 140, 1754-1764. [CrossRef]

4. Mokhlis, N.A.; Ibrahim, E.J.; Ibrahim, E.J. Stress-strength reliability with general form distributions. Commun.-Stat.-Theory Methods 2017, 46, 1230-1246. [CrossRef]

5. Wang, B.; Geng, Y.; Zhou, J. Inference for the generalized exponential stress-strength model. Appl. Math. Model. 2018, 53, 267-275. [CrossRef]

6. Bhattacharyya, G.K.; Johnson, R.A. Estimation of reliability in multicomponent stresss-trength model. J. Am. Stat. Ass. 1974, 69, 966-970. [CrossRef]

7. Dey, S.; Mazucheli, J.; Anis, M.Z. Estimation of reliability of multicomponent stress-strength for a Kumaraswamy distribution. Commun.-Stat.-Theory Methods 2017, 46, 1560-1572. [CrossRef]

8. Kayal, T.; Tripathi, Y.M.; Dey, S.; Wu, S.J. On estimating the reliability in a multicomponent stress-strength model based on Chen distribution. Commun.-Stat.-Theory Methods 2020, 49, 2429-2447. [CrossRef]

9. Kizilaslan, F. Classical and Bayesian estimation of reliability in a multicomponent stress-strength model based on a general class of inverse exponentiated distributions. Stat. Pap. 2018, 59, 1161-1192. [CrossRef]

10. Kizilaslan, F. Classical and Bayesian estimation of reliability in a multicomponent stress-strength model based on the proportional reversed hazard rate model. Math. Comput. Sim. 2017, 136, 36-62. [CrossRef]

11. Kizilaslan, F.; Nadar, M. Estimation of reliability in a multicomponent stress-strength model based on a bivariate Kumaraswamy distribution. Stat. Pap. 2018, 59, 307-340. [CrossRef]

12. Nadar, M.; Kizilaslan, F. Estimation of reliability in a multicomponent stress-strength model based on a Marshall-Olkin bivariate Weibull distribution. IEEE Trans. Reliab. 2016, 65, 370-380. [CrossRef]

13. Rao, G.S.; Aslam, M.; Aslam, M. Burr type XII distribution parametric estimation and estimation of reliability of multicomponent stress-strength. Commun.-Stat.-Theory Methods 2015, 44, 4953-4961. [CrossRef]

14. Rayleigh, J.W.S. On the resultant of a large number of vibrations of the some pitch and of arbitrary phase. Phil. Mag. 1880, 10, 73-78. [CrossRef]

15. Ahrari, V.; Baratpour, S.; Habibirad, A.; Fakoor, V. Goodness of fit tests for Rayleigh distribution based on quantiles. Commun.Stat.-Simul. Comput. 2019, [CrossRef]

16. Dey, T.; Dey, S.; Kundu, D. On progressively Type-II censored two-parameter Rayleigh distribution. Commun.-Stat.-Simul. Comput. 2016, 45, 438-455. [CrossRef]

17. Fundi, M.D.; Njenga, E.G.; Keitany, K.G. Estimation of parameters of the two parameter Rayleigh distribution based on progressive Type-II censoring using maximum likelihood method via the NR and the EM algorithms. Am. J. Theor. Appl. Stat. 2017, 6, 1-9. [CrossRef]

18. Ghani, A.S.A.; Isa, N.A.M. Underwater image quality enhancement through integrated color model with Rayleigh distribution. Appl. Soft. Comput. 2015, 27, 219-230. [CrossRef]

19. Rao, G.S. Estimation of reliability in multicomponent stress-strength model based on Rayleigh distribution. ProbStat Forum. 2012, $5,150-161$.

20. Weerahandi, S. Generalized Inference in Repeated Measures: Exact Methods in MANOVA and Mixed Models; Wiley: New York, NY, USA, 2004.

21. $\mathrm{Xu}, \mathrm{J} . ;$ Long, J.S. Using the delta method tonconstruct confidence intervals for predicted probabilities, rates, and discrete changes, In Lecture Notes; Indiana University: Bloomington, IN, USA, 2005.

22. Lawless, J.F. Statistical Models and Methods for Lifetime Data, 2nd ed.; Wiley: New York, NY, USA, 2003.

23. Stephens, M.A. Tests for the exponential distribution. In Goodness-of-Fit Techniques; D'Agostino, R.B., Stephens, M.A., Eds.; Marcel Dekker: New York, NY, USA, 1986; pp. 421-459.

24. Viveros, R.; Balakrishnan, N. Interval estimation of parameters of life from progressively censored data. Technometrics 1994, 36, 84-91. [CrossRef] 